\title{
Combinatorics of Triangulations and the Chern-Simons Invariant for Hyperbolic 3-Manifolds
}

\author{
Walter D. Neumann
}

\section{Introduction}

In this paper we prove some results on combinatorics of triangulations of 3-dimensional pseudo-manifolds, improving on results of [NZ], and apply them to obtain a simplicial formula for the Chern-Simons invariant of an ideally triangulated hyperbolic 3-manifold. Combining this with [MN] gives a simplicial formula for the $\eta$ invariant also.

In effect, the main ingredient in the formula is the sum of the "Rogers dilogarithm" of the complex parameters of the ideal tetrahedra of the triangulation, but the choice of the appropriate branch of the Rogers dilogarithm for each simplex involves unexpected combinatorics (cf. Remark 4 below for this interpretation of the formula).

The combinatorial part of this paper (Sects. 4-6) is self-contained and of independent interest. For instance, T. Yoshida [Y2] has used these combinatorics (in the version of [NZ]) to study character varieties and boundary slopes in the spirit of Culler-Shalen [CS].

In the remainder of this Introduction we summarize the application to the ChernSimons invariant. All manifolds in this paper are assumed to be oriented.

If $M$ is a complete hyperbolic 3-manifold which is compact, then its Chern-Simons invariant $\operatorname{CS}(M)$ is well-defined modulo $2 \pi^{2}$. If $M$ is non-compact then Bob Meyerhoff has shown in $[\mathbf{M}]$ that there is still a natural definition of $\operatorname{CS}(M)$ which is well-defined modulo $\pi^{2}$. Let $\mathcal{V}(M)=\operatorname{Vol}(M)+i \operatorname{CS}(M)$, which is well-defined modulo $i 2 \pi^{2} \mathbb{Z}$ or $i \pi^{2} \mathbb{Z}$.

A formula for $\mathcal{V}\left(M^{\prime}\right) \bmod i \pi^{2} \mathbb{Z}$, as $M^{\prime}$ varies over the hyperbolic Dehn surgeries on $M$, was conjectured in [NZ] and proved by T. Yoshida in [Y1]. This formula is of theoretical interest but is not practical for actually computing $\mathcal{V}\left(M^{\prime}\right)$. It is not hard to reverse the derivation in [NZ] to obtain a computable formula in terms of an ideal triangulation of $M$. However, the resulting formula involves an unknown constant which depends on the combinatorics of the triangulation of $M$ and which seems hard to determine in general. Using a result of Dupont [D] we can find a version of the formula in which this constant is at least a rational multiple of $i \pi^{2}$ (Theorem 1 below). Using a more careful analysis of the relevant combinatorics we are able to give a version (Theorem 2) in which the constant is conjecturally in $\left(i \pi^{2} / 6\right) \mathbb{Z}$ and is thus determined up to a six-fold ambiguity (since it lives in $\mathbb{C} \bmod i \pi^{2} \mathbb{Z}$ ).

Suppose $M$ has an ideal triangulation which subdivides it into $n$ ideal tetrahedra

$$
M=\Delta_{1} \cup \ldots \cup \Delta_{n} .
$$

Choose an edge of the $j$-th tetrahedron and let $z_{j}^{0}$ be the complex parameter which then describes this tetrahedron (we are following the notation of [NZ] which may be consulted 
for more details - see also Sect. 2). Let

$$
z^{0}=\left(\begin{array}{c}
\log z_{1}^{0} \\
\vdots \\
\log z_{n}^{0} \\
\log \left(1-z_{1}^{0}\right) \\
\vdots \\
\log \left(1-z_{n}^{0}\right)
\end{array}\right)
$$

Recall from [NZ] (see also Sect. 2) that if $M$ has $h$ cusps then the $z_{j}^{0}$ are determined by so-called consistency and cusp relations which can be written in the form

$$
U Z^{0}=\pi i \mathbf{d}
$$

where $U$ is a certain integral $(n+2 h) \times 2 n$-matrix and

$$
\mathbf{d}=\left(\begin{array}{c}
d_{1} \\
\vdots \\
d_{n+2 h}
\end{array}\right)
$$

is some integral vector. The equation

$$
U \mathbf{c}=\mathbf{d}
$$

has a solution $\mathbf{c}=Z^{0} / \pi i \in \mathbb{C}^{2 n}$. Since $U$ is an integral matrix, (*) also has solutions

$$
\mathbf{c}=\left(\begin{array}{c}
c_{1}^{\prime} \\
\vdots \\
c_{n}^{\prime} \\
c_{1}^{\prime \prime} \\
\vdots \\
c_{n}^{\prime \prime}
\end{array}\right) \in \mathbb{Q}^{2 n}
$$

We shall see that solutions $\mathbf{c}$ can be found in $\mathbb{Z}^{2 n}$, in fact even in a certain sublattice of $\mathbb{Z}^{2 n}$.

Let $M^{\prime}$ be the result of a hyperbolic Dehn surgery on $M$ obtained by deforming the parameters $z_{j}^{0}$ to new values $z_{j}$ (cf. e.g., [NZ]). Topologically $M^{\prime}$ differs from $M$ in that a new closed geodesic $\gamma_{j}$ has been added at the $j$-th cusp for some $j \in\{1, \ldots, h\}$. Let $\lambda_{j}$ be the complex number which has real part equal to the length of this geodesic and imaginary part equal to its torsion (the latter is only well-defined modulo $2 \pi$ ). If no geodesic has been added at the $j$-th cusp we put $\lambda_{j}=0$.

Let $\mathcal{R}(z)$ be the "Rogers dilogarithm function," which is related to the usual dilogarithm $\mathrm{Li}_{2}$ by

$$
\begin{aligned}
\mathcal{R}(z) & =\frac{1}{2} \log (z) \log (1-z)+\operatorname{Li}_{2}(z) \\
& =\frac{1}{2} \log (z) \log (1-z)-\int_{0}^{z} \frac{\log (1-t)}{t} d t .
\end{aligned}
$$


Theorem 1. Given any solution $\mathbf{c} \in \mathbb{C}^{2 n}$ to $(*)$, there exists a constant $\alpha=\alpha(\mathbf{c})$ such that if $M^{\prime}$ is the result of hyperbolic Dehn surgery on $M$, then

$$
\mathcal{V}\left(M^{\prime}\right)=\alpha-\frac{\pi}{2} \sum_{j=1}^{h} \lambda_{j}-i \sum_{\nu=1}^{n}\left(\mathcal{R}\left(z_{\nu}\right)-\frac{i \pi}{2}\left(\overline{c_{\nu}^{\prime}} \log \left(1-z_{\nu}\right)-\overline{c_{\nu}^{\prime \prime}} \log \left(z_{\nu}\right)\right)\right) .
$$

Moreover, if $\mathbf{c} \in \mathbb{R}^{2 n}$ then $\alpha$ is pure imaginary and if $\mathbf{c} \in \mathbb{Q}^{2 n}$ then $\alpha \in i \pi^{2} \mathbb{Q}$.

In Sect. 2 we will define a "parity condition" on $\mathbf{c} \in \mathbb{Z}^{2 n}$ which depends on the combinatorics of our situation.

Theorem 2. There exist solutions $\mathbf{c}$ to $(*)$ in $\mathbb{Z}^{2 n}$ satisfying the parity condition. For such $\mathbf{c}$, the constant $\alpha$ of Theorem 1 is well-defined (i.e., independent of $\mathbf{c}$ ) modulo $i \pi^{2} / 2$ (and is conjecturally an integer multiple of $i \pi^{2} / 6$ ).

The formula of Theorem 1 is a fairly easy application of the methods in [NZ]; we prove it in Sect. 3, along with the rationality statement for $\alpha$ and the second sentence of Theorem 2. The existence of $\mathbf{c}$ as in Theorem 2 needs the combinatorial results of Sects. $4-6$ and is proved in Sect. 6.

Remarks. 1. If one drops the parity condition in Theorem 2 then $\alpha$ is well-defined modulo $i \pi^{2} / 4$ instead of $i \pi^{2} / 2$ and is conjecturally an integral multiple of $i \pi^{2} / 12$.

2. Denote $S_{0 l}=\left\{\mathbf{c} \in \mathbb{Z}^{2 n}: U \mathbf{c}=\mathbf{d}\right\}$ and $S o l=\left\{\mathbf{c} \in S o l_{0}: \mathbf{c}\right.$ satisfies the parity condition $\}$. It follows from Theorem 4.2 that $S_{0} / S o l \cong H^{1}\left(M^{*} ; \mathbb{Z} / 2\right)=$ $\operatorname{Hom}(\Gamma / P, \mathbb{Z} / 2)$, where $M^{*}$ is the end compactification of $M$ and $P$ is the subgroup of $\Gamma=\pi_{1} M$ generated by all parabolic elements of $\Gamma$. In particular, Sol $=S_{0} l_{0}$ if $\Gamma / P$ has odd order.

3. Our formula has been implemented in Jeff Weeks' program "snap pea" by R. Meyerhoff and C. Hodgson, using c in $S_{0} l_{0}$ rather than Sol (see [HMW] for a brief description of this program). There is therefore now a large accumulation of experimental evidence for the $i \pi^{2} / 12$ conjecture of Remark 1 above. In cases when $S o l=S o l_{0}$ the computations appear to confirm the stronger $i \pi^{2} / 6$ conjecture, but I have not checked systematically.

4. Note that $\mathcal{R}(z)$ is a multivalued function of $z$. It has singularities at 0 and 1 and is single-valued on the universal cover of $\mathbb{C}-\{0,1\}$. We only need $\mathcal{R}(z)$ $\left(\bmod \pi^{2}\right)$, and in fact $\mathcal{R}(z)\left(\bmod 2 \pi^{2}\right)$ is well-defined on the universal abelian cover $X$ of $\mathbb{C}-\{0,1\}$ ( $\mathcal{R}$ itself is well-defined on the universal nilpotent cover). A point of $X$ is determined by a point $z$ of a fundamental domain for the cover and an integer pair $\left(c^{\prime}, c^{\prime \prime}\right) \in \mathbb{Z}^{2}=\pi_{1}(\mathbb{C}-\{0,1\})$. The value of $\mathcal{R} \bmod 2 \pi^{2}$ at this point is then

$$
\mathcal{R}(z)+i \pi\left(c^{\prime} \log (1-z)-c^{\prime \prime} \log (z)\right),
$$

where $\mathcal{R}(z)$ is the standard branch on the fundamental domain. Thus, when $\mathbf{c}$ is integral, the summand

$$
\mathcal{R}\left(z_{\nu}\right)-\frac{i \pi}{2}\left(\overline{c_{\nu}^{\prime}} \log \left(1-z_{\nu}\right)-\overline{c_{\nu}^{\prime \prime}} \log \left(z_{\nu}\right)\right) \quad\left(\bmod \pi^{2}\right)
$$


in our formula can be thought of as representing a branch at $z_{\nu}$ of one of the four functions

$$
\begin{gathered}
\mathcal{R}(z), \quad \mathcal{R}(z)+\frac{i \pi}{2} \log (z), \quad \mathcal{R}(z)+\frac{i \pi}{2} \log (1-z), \quad \text { or } \\
\mathcal{R}(z)+\frac{i \pi}{2}(\log (1-z)+\log (z)) \quad\left(\bmod \pi^{2}\right),
\end{gathered}
$$

according to the parity of $c_{\nu}^{\prime}$ and $c_{\nu}^{\prime \prime}$. Each of the first three of these functions can be naturally associated to the choice of an edge of the ideal tetrahedron with parameter $z$, since choosing a different edge replaces $z$ by $1-1 / z$ or $1 /(1-z)$ and $\mathcal{R}(z)$ satisfies the functional equations

$$
\begin{aligned}
& \mathcal{R}\left(1-\frac{1}{z}\right)=\mathcal{R}(z)+\frac{i \pi}{2} \log (z)-\frac{\pi^{2}}{6} \\
& \mathcal{R}\left(\frac{1}{1-z}\right)=\mathcal{R}(z)+\frac{i \pi}{2} \log (1-z)+\frac{\pi^{2}}{6} .
\end{aligned}
$$

The last of the above four functions is mapped to a different branch of itself, up to a constant, by the transformation $z \mapsto(1-1 / z)$.

5 . We believe there should be a topological interpretation of an integer vector $\mathbf{c}$ as in Theorem 2: it should be associated with some kind of extra structure on $M$. Remark 4 and Section 2 both give some support for this belief.

Acknowledgements. The support of the N.S.F. for this work is gratefully acknowledged. Don Zagier encouraged me to finally write up this work, despite the lack of proof of the conjecture of Theorem 2 .

\section{Consistency and cusp conditions, and the conditions on $\mathrm{c}$}

An ideal hyperbolic structure on a tetrahedron $\Delta$ is specified by assigning a complex number with positive imaginary part to each edge, these numbers being related as in Fig. 1. The geometric meaning of these parameters is that the Euclidean triangle cut off by a horosphere section at a vertex is similar to the Euclidean triangle pictured in Fig. 1 with vertices 0,1 , and $z$ in the complex plane; the parameter $z$ is only well-defined after choosing an edge of $\Delta$, and parameters for different edges are related as illustrated. Instead of these parameters $z, 1 /(1-z)$, and $(z-1) / z$, it will be convenient to use their natural logarithms

$$
\log (z), \quad-\log (1-z), \quad \log (1-z)-\log (z)+\pi i
$$

(unless otherwise stated, log is always the standard branch on $\mathbb{C}-(-\infty, 0])$. We call these the log-parameters of the tetrahedron.

Given a tetrahedron $\Delta$, we shall choose a labelling of its edges $e_{1}, e_{2}, \ldots, e_{6}$ as in Fig. 2. Thus $e_{j}$ and $e_{j+3}$ are opposite edges for $j=1,2,3$.

Let $M$ be a hyperbolic 3-manifold which is ideally triangulated as

$$
M=\Delta_{1} \cup \ldots \cup \Delta_{n}
$$

Choose a labelling $e_{j}, j=1, \ldots, 6$, of the edges of each $\Delta_{\nu}$ as above. Let $z_{\nu}$ be the parameter for $\Delta_{\nu}$ with respect to the edge $e_{1}$ of $\Delta_{\nu}$. We do not assume the hyperbolic 


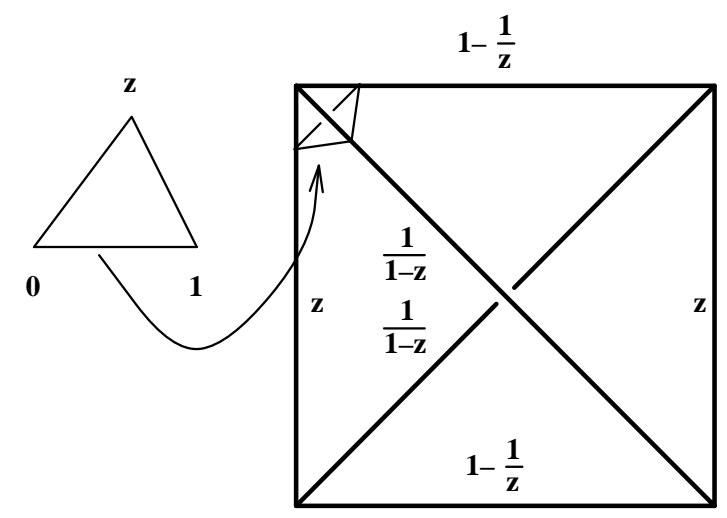

Figure 1

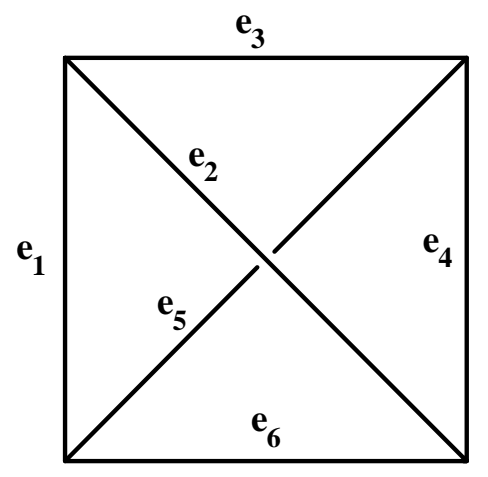

Figure 2

structure on $M$ is complete, but we assume it results by deforming from a complete structure on $M$ with tetrahedral parameters $z_{\nu}^{0}$ say.

Consistency conditions. Each edge of each tetrahedron of the triangulation of $M$ has an associated log-parameter. The condition that the ideal tetrahedra fit together around an edge $E$ of the triangulation is equivalent to the condition that the log-parameters sum to $2 \pi i$ around this edge. We call this the consistency condition at the edge $E$. It has the form

$$
\sum_{\nu=1}^{n} r_{\nu}^{\prime}(E) \log \left(z_{\nu}\right)+r_{\nu}^{\prime \prime}(E) \log \left(1-z_{\nu}\right)=d(E) \pi i
$$

for some integer $d(E)$ and integers $r_{\nu}^{\prime}(E)$ and $r_{\nu}^{\prime \prime}(E),(\nu=1, \ldots, n)$. 
We can describe the integer $d(E)$ as follows. Associate an integer $\epsilon_{i}\left(\Delta_{\nu}\right)$ to the $i$-th edge of $\Delta_{\nu}$ as follows:

$$
\begin{aligned}
& \epsilon_{1}\left(\Delta_{\nu}\right)=\epsilon_{4}\left(\Delta_{\nu}\right)=0, \\
& \epsilon_{2}\left(\Delta_{\nu}\right)=\epsilon_{5}\left(\Delta_{\nu}\right)=0, \\
& \epsilon_{3}\left(\Delta_{\nu}\right)=\epsilon_{6}\left(\Delta_{\nu}\right)=1 .
\end{aligned}
$$

Associate a complex number $\zeta_{j}\left(\Delta_{\nu}\right)$ to the $j$-th edge of $\Delta_{\nu}$ by:

$$
\begin{aligned}
& \zeta_{1}\left(\Delta_{\nu}\right)=\zeta_{4}\left(\Delta_{\nu}\right)=\log \left(z_{\nu}\right), \\
& \zeta_{2}\left(\Delta_{\nu}\right)=\zeta_{5}\left(\Delta_{\nu}\right)=-\log \left(1-z_{\nu}\right), \\
& \zeta_{3}\left(\Delta_{\nu}\right)=\zeta_{6}\left(\Delta_{\nu}\right)=-\log \left(z_{\nu}\right)+\log \left(1-z_{\nu}\right) .
\end{aligned}
$$

The log-parameters of $\Delta_{\nu}$ are then

$$
\mu_{j}\left(\Delta_{\nu}\right)=\zeta_{j}\left(\Delta_{\nu}\right)+\epsilon_{j}\left(\Delta_{\nu}\right) \pi i, \quad j=1, \ldots, 6 .
$$

Equation (2.2) is the result of equating the sum of log-parameters around the edge $E$ with $2 \pi i$ and moving the $\epsilon_{j}\left(\Delta_{\nu}\right) \pi i$ terms to the right of the equation. Thus

Lemma 2.1. $d(E)=2-\sum_{\kappa} \epsilon_{j_{\kappa}}\left(\Delta_{\nu_{\kappa}}\right)$ where the sum is the sum of the $\epsilon_{j}\left(\Delta_{\nu}\right)$ around the edge $E$.

Cusp conditions. The torus $T$ section of an end of $M$ is triangulated by triangles cut off the vertices of the $\Delta_{\nu}$ by horospheres. Each vertex of a triangle of this triangulation determines some edge of a tetrahedron, and hence has an associated log-parameter, which can be written $\mu=\zeta+\epsilon \pi i$ as in equation (2.5).

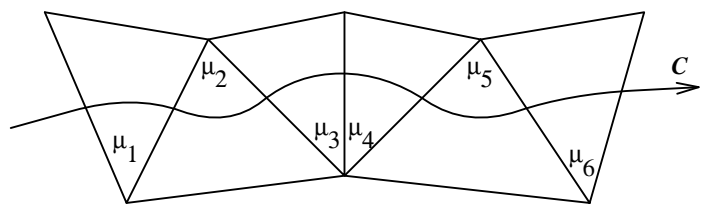

$$
\begin{gathered}
\mu(C)=-\mu_{1}+\mu_{2}- \\
\mu_{3}-\mu_{4}+\mu_{5}- \\
\mu_{6}+\ldots
\end{gathered}
$$

Figure 3

Let $C$ be an essential simple closed curve in $T$ which is in general position with respect to the 1-skeleton of the triangulation of $T$ and, moreover, "has no back-tracking," in the sense that it never departs a 2 -simplex across the same edge by which it entered. Then as $C$ passes through a 2 -simplex it determines a vertex of the 2-simplex, the vertex between the entering and departing edges, and a sign + or - according as it goes past this vertex counter-clockwise or clockwise. Let $\mu(C)=\sum \pm \mu_{j}$ be the sum over the vertices that $C$ passes of the corresponding log-parameter $\mu_{j}$ with sign \pm as above (Fig. 3). Clearly,

$$
\mu(C)=\sum_{\nu=1}^{n} r_{\nu}^{\prime}(C) \log \left(z_{\nu}\right)+r_{\nu}^{\prime \prime}(C) \log \left(1-z_{\nu}\right)-d(C) \pi i
$$

for some integer $d(C)$ and integers $r_{\nu}^{\prime}(C)$ and $r_{\nu}^{\prime \prime}(C),(\nu=1, \ldots, n)$. 
It is not hard to see (cf. Sect. 6 or [NZ]) that, given the consistency conditions, $\mu(C)$ only depends on the homology class of $C$ in $T$ and $\mu$ gives a homomorphism from $H_{1}(T)$ to $\mathbb{C}$. In fact, $\mu(C)=2 \log \lambda(H(C))$, where $\lambda(H(C))$ is an eigenvalue of the holonomy $H(C)$ of $C$.

The cusp condition corresponding to $C$ is the condition that $C$ have parabolic holonomy, that is, $\mu(C)=0$, which by (2.6) can be written

$$
\sum_{\nu=1}^{n} r_{\nu}^{\prime}(C) \log \left(z_{\nu}\right)+r_{\nu}^{\prime \prime}(C) \log \left(1-z_{\nu}\right)=d(C) \pi i
$$

Just as for Lemma 2.1 one sees:

Lemma 2.2. $d(C)=-\sum \pm \epsilon_{j}$, where the $\epsilon_{j}$ are the $\epsilon$ 's (cf. equation (2.3)) at the vertices that $C$ passes, with signs \pm given as above.

Consistency and cusp conditions. For each edge $E$ of our triangulation we have the consistency condition (2.2). At each cusp of $M$ choose two curves $C$ as above representing a basis of the first homology of the cusp and consider the corresponding cusp conditions (2.7). The complete hyperbolic structure on $M$ - the one with tetrahedral parameters $z_{\nu}^{0}$-is determined by all of these consistency and cusp conditions together. This is a system of linear equations with integral coefficients, which can be written

$$
U Z^{0}=\pi i \mathbf{d}
$$

as described in the Introduction, with

$$
z^{0}=\left(\begin{array}{c}
\log z_{1}^{0} \\
\vdots \\
\log z_{n}^{0} \\
\log \left(1-z_{1}^{0}\right) \\
\vdots \\
\log \left(1-z_{n}^{0}\right)
\end{array}\right) .
$$

Now suppose we are given a $2 n$-vector $\mathbf{c}=\left(c_{1}^{\prime}, \ldots, c_{n}^{\prime}, c_{1}^{\prime \prime}, \ldots, c_{n}^{\prime \prime}\right)^{\mathrm{t}}$. Assign numbers $\eta_{i}\left(\Delta_{\nu}\right)$ to the edges of $\Delta_{\nu}$ as follows:

$$
\begin{aligned}
& \eta_{1}\left(\Delta_{\nu}\right)=\eta_{4}\left(\Delta_{\nu}\right)=c_{\nu}^{\prime}, \\
& \eta_{2}\left(\Delta_{\nu}\right)=\eta_{5}\left(\Delta_{\nu}\right)=-c_{\nu}^{\prime \prime}, \\
& \eta_{4}\left(\Delta_{\nu}\right)=\eta_{6}\left(\Delta_{\nu}\right)=-c_{\nu}^{\prime}+c_{\nu}^{\prime \prime}+1 .
\end{aligned}
$$

Proposition 2.3. The equation $U \mathbf{c}=\mathbf{d}$ (equation (*) of the Introduction) is equivalent to the following conditions:

C1. the sum of the $\eta$ 's around any edge $E$ of the triangulation is 2;

C2. for any path $C$ as above in a torus section of an end of $M$, the signed sum of the $\eta$ 's over the vertices adjacent to the path $C$ is zero.

Proof. Replace $\log \left(z_{\nu}^{0}\right)$ by $c_{\nu}^{\prime}$ and $\log \left(1-z_{\nu}^{0}\right)$ by $c_{\nu}^{\prime \prime}$ in the proofs of Lemmas 2.1 and 2.2. 
The parity conditions. We consider an integral vector $\mathbf{c} \in \mathbb{Z}^{2 n}$. Thus, the $\eta_{j}\left(\Delta_{\nu}\right)$ defined above are integers. Let $C$ be any closed path in $M$ which is in general position with respect to the 2 -skeleton of our triangulation. We also assume $C$ "has no backtracking" in the sense that it never departs a 3-simplex across the same face by which it entered. Then as $C$ passes through a 3-simplex it determines an edge of the 3-simplex the edge common to the entering and departing faces - and hence an integer $\eta$ as above. We call the modulo-2 sum of these $\eta$ 's along $C$ the parity of $\mathbf{c}$ along $C$.

The parity condition on $\mathbf{c}$ is the condition:

C3. The parity of $\mathbf{c}$ along $C$ is even for all $C$ as above.

The combinatorial part of Theorem 2 is re-formulated and made precise in part (i) of the following theorem, which will be proved in Sect. 6 .

\section{Theorem 2.4.}

(i) There exists $\mathbf{c} \in \mathbb{Z}^{2 n}$ satisfying the above conditions $\mathrm{C} 1, \mathrm{C} 2$, and $\mathrm{C} 3$.

(ii) Any two elements $\mathbf{c}_{1}$ and $\mathbf{c}_{2}$ of $\mathbb{Z}^{2 n}$ satisfying conditions $\mathrm{C} 1, \mathrm{C} 2$, and $\mathrm{C} 3$, differ by an integral linear combination of the vectors

$$
J_{2 n} \mathbf{r}(E)=\left(r_{1}^{\prime \prime}(E), \ldots, r_{n}^{\prime \prime}(E),-r_{1}^{\prime}(E), \ldots,-r_{n}^{\prime}(E)\right)^{\mathrm{t}},
$$

where the $\mathbf{r}(E)$ are the coefficient vectors of the consistency conditions and

$$
J_{2 n}=\left(\begin{array}{cc}
0 & I_{n} \\
-I_{n} & 0
\end{array}\right)
$$

Remark. The parity condition $\mathrm{C} 3$ implies that $\mathrm{C} 1$ holds modulo 2 . Given that $\mathrm{C} 1$ holds modulo 2, the parity of $\mathbf{c}$ along $C$ is easily seen to depend only on the homology class of $C$ and to give a homomorphism $H_{1}(M ; \mathbb{Z} / 2) \rightarrow \mathbb{Z} / 2$. If $\mathrm{C} 2$ holds modulo 2 , this homomorphism vanishes on $H_{1}(\mathcal{E} ; \mathbb{Z} / 2)$, where $\mathcal{E}$ is the union of the ends of $M$. Thus, one then need only check the parity condition on a set of representatives for generators of $H_{1}(M ; \mathbb{Z} / 2) / \operatorname{Im} H_{1}(\mathcal{E} ; \mathbb{Z} / 2)=H_{1}\left(M^{*} ; \mathbb{Z} / 2\right)$, where $M^{*}$ is the end compactification of $M$.

\section{The formula for the Chern-Simons invariant}

In this section we prove Theorems 1 and 2 of the Introduction, assuming Theorem 2.4, which will be proved in Sect. 6 .

Proof of Theorem 1. Let $M$ be triangulated, as in the previous section, into ideal tetrahedra with parameters $z_{\nu}$. We do not assume $M$ has the complete hyperbolic structure. Under certain conditions that we recall below, the metric completion $M^{\prime}$ of this structure on $M$ is a hyperbolic manifold, in which case it is a Dehn filling of $M$ in the topological sense. To review the conditions we need some notation.

Number the ends of $M$ by $j=1, \ldots, h$ say. Choose a specific oriented basis $\mathbf{l}_{j}$, $\mathbf{m}_{j}$ for the homology of the $j$-th end of $M$ for each $j$, and let

$$
u_{j}=\mu\left(\mathbf{m}_{j}\right), \quad v_{j}=\mu\left(\mathbf{l}_{j}\right), \quad(j=1, \ldots, h),
$$

where $\mu\left(\mathbf{m}_{j}\right)$ means $\mu(C)$ for some curve $C$ representing $\mathbf{m}_{j}$, and the same for $\mu\left(\mathbf{l}_{\mathbf{j}}\right)$. 
If $u_{j}=v_{j}=0$ then $M$ is still complete at the $j$-th end, that is, it has a cusp there. If coprime integers $p_{j}$ and $q_{j}$ exist with

$$
p_{j} u_{j}+q_{j} v_{j}=2 \pi i,
$$

then $M^{\prime}$ is a hyperbolic manifold near the $j$-th end of $M$, obtained by adding a geodesic $\gamma_{j}$ to $M$ there in such a way that it is topologically a $\left(p_{j}, q_{j}\right)$-Dehn filling, that is, a Dehn filling which kills the homology class $p_{j} \mathbf{m}_{j}+q_{j} \mathbf{l}_{j}$. In all other cases $M^{\prime}$ is not a hyperbolic manifold at the $j$-th end of $M$.

Remark on orientations. In [NZ] $\left\{\mathbf{m}_{j}, \mathbf{l}_{j}\right\}$ was an oriented basis rather than $\left\{\mathbf{l}_{j}, \mathbf{m}_{j}\right\}$. For the justification of the different convention used here, see the "Note on orientations" in section 6 of [NR] (these Proceedings) or in [MN].

Assume now that $M^{\prime}$ is a hyperbolic manifold. Let $\lambda_{j}$ be as in the Introduction: $\lambda_{j}=0$ if the $j$-th end of $M$ is complete, and otherwise $\lambda_{j}$ is the "complex length" of the added geodesic $\gamma_{j}$, that is, its length plus $i$ times its torsion.

Lemma 3.1. $\operatorname{Re} \lambda_{j}=-\frac{1}{2 \pi} \operatorname{Im}\left(v_{j} \overline{u_{j}}\right)$.

Proof. This is Proposition 4.3 of [NZ], except that $u_{j}$ and $v_{j}$ have been interchanged, in keeping with the above orientation convention.

Following equation (2.6), we can write

$$
\begin{aligned}
& u_{j}=\sum_{\nu=1}^{n}\left(r_{\nu}^{\prime}\left(\mathbf{m}_{j}\right) \log \left(z_{\nu}\right)+r_{\nu}^{\prime \prime}\left(\mathbf{m}_{j}\right) \log \left(1-z_{\nu}\right)\right)-d\left(\mathbf{m}_{j}\right) \pi i \\
& v_{j}=\sum_{\nu=1}^{n}\left(r_{\nu}^{\prime}\left(\mathbf{l}_{j}\right) \log \left(z_{\nu}\right)+r_{\nu}^{\prime \prime}\left(\mathbf{l}_{j}\right) \log \left(1-z_{\nu}\right)\right)-d\left(\mathbf{l}_{j}\right) \pi i
\end{aligned}
$$

Given a solution $\mathbf{c}=\left(c_{1}^{\prime}, \ldots, c_{n}^{\prime}, c_{1}^{\prime \prime}, \ldots, c_{n}^{\prime \prime}\right)^{\mathrm{t}}$ to the equation $U \mathbf{c}=\mathbf{d}$, we can rewrite this

$$
\begin{aligned}
& u_{j}=\sum_{\nu=1}^{n}\left(r_{\nu}^{\prime}\left(\mathbf{m}_{j}\right)\left(\log \left(z_{\nu}\right)-c_{\nu}^{\prime} \pi i\right)+r_{\nu}^{\prime \prime}\left(\mathbf{m}_{j}\right)\left(\log \left(1-z_{\nu}\right)-c_{\nu}^{\prime \prime} \pi i\right)\right) \\
& v_{j}=\sum_{\nu=1}^{n}\left(r_{\nu}^{\prime}\left(\mathbf{l}_{j}\right)\left(\log \left(z_{\nu}\right)-c_{\nu}^{\prime} \pi i\right)+r_{\nu}^{\prime \prime}\left(\mathbf{l}_{j}\right)\left(\log \left(1-z_{\nu}\right)-c_{\nu}^{\prime \prime} \pi i\right)\right) .
\end{aligned}
$$

As in [NZ], we write the matrix $U$ as

$$
U=\left(\begin{array}{c}
M \\
L \\
R
\end{array}\right)=\left(\begin{array}{l}
C \\
R
\end{array}\right),
$$


where the rows of $R$ are the coefficients of the consistency conditions and

$$
\begin{aligned}
L & =\left(\begin{array}{cccccc}
r_{1}^{\prime}\left(\mathbf{l}_{1}\right) & \ldots & r_{n}^{\prime}\left(\mathbf{l}_{1}\right) & r_{1}^{\prime \prime}\left(\mathbf{l}_{1}\right) & \ldots & r_{n}^{\prime \prime}\left(\mathbf{l}_{1}\right) \\
\vdots & & \vdots & \vdots & & \vdots \\
r_{1}^{\prime}\left(\mathbf{l}_{h}\right) & \ldots & r_{n}^{\prime}\left(\mathbf{l}_{h}\right) & r_{1}^{\prime \prime}\left(\mathbf{l}_{h}\right) & \ldots & r_{n}^{\prime \prime}\left(\mathbf{l}_{h}\right)
\end{array}\right) \\
M & =\left(\begin{array}{cccccc}
r_{1}^{\prime}\left(\mathbf{m}_{1}\right) & \ldots & r_{n}^{\prime}\left(\mathbf{m}_{1}\right) & r_{1}^{\prime \prime}\left(\mathbf{m}_{1}\right) & \ldots & r_{n}^{\prime \prime}\left(\mathbf{m}_{1}\right) \\
\vdots & & \vdots & \vdots & & \vdots \\
r_{1}^{\prime}\left(\mathbf{m}_{h}\right) & \ldots & r_{n}^{\prime}\left(\mathbf{m}_{h}\right) & r_{1}^{\prime \prime}\left(\mathbf{m}_{h}\right) & \ldots & r_{n}^{\prime \prime}\left(\mathbf{m}_{h}\right)
\end{array}\right)
\end{aligned}
$$

We also write

$$
z=\left(\begin{array}{c}
\log z_{1} \\
\vdots \\
\log z_{n} \\
\log \left(1-z_{1}\right) \\
\vdots \\
\log \left(1-z_{n}\right)
\end{array}\right)
$$

Then equations (3.1) can be written in matrix form:

$$
\left(\begin{array}{c}
u_{1} \\
\vdots \\
u_{n} \\
v_{1} \\
\vdots \\
v_{n}
\end{array}\right)=C(z-\mathbf{c} \pi i)
$$

and the consistency relations can be written

$$
R(z-\mathbf{c} \pi i)=0 .
$$

We recall the main combinatorial lemma of [NZ]. Let $J_{2 m}$ denote the $2 m \times 2 m$ matrix

$$
J_{2 m}=\left(\begin{array}{cc}
0 & I \\
-I & 0
\end{array}\right)
$$

Lemma 3.2. Given $\mathrm{x}$ and $\mathrm{y}$ in $\mathbb{C}^{2 n}$ satisfying $R \mathbf{x}=R \mathbf{y}=0$, we have

$$
\mathbf{x}^{\mathrm{t}} J_{2 n} \overline{\mathbf{y}}=\frac{1}{2} \mathbf{x}^{\mathrm{t}} C^{\mathrm{t}} J_{2 h} C \overline{\mathbf{y}} .
$$

Proof. This is Corollary 2.4 of [NZ], modified in accordance with our different orientation convention. It also follows easily from Theorem 4.1 below. 
We can apply this lemma with $\mathbf{x}=\mathbf{y}=Z-\mathbf{c} \pi i$ to get

$$
\begin{aligned}
\sum_{j=1}^{h} \operatorname{Im}\left(v_{j} \bar{u}_{j}\right)= & \frac{i}{2}\left(u_{1}, \ldots, v_{h}\right) J_{2 h}\left(\begin{array}{c}
\bar{u}_{1} \\
\vdots \\
\bar{v}_{h}
\end{array}\right) \\
= & \frac{i}{2}(z-\mathbf{c} \pi i)^{\mathrm{t}} C^{\mathrm{t}} J_{2 h} C(\overline{\bar{z}}+\overline{\mathbf{c}} \pi i) \\
= & i(z-\mathbf{c} \pi i)^{\mathrm{t}} J_{2 n}(\bar{z}+\overline{\mathbf{c}} \pi i) \\
= & -2 \sum_{\nu=1}^{n} \operatorname{Im}\left(\log z_{\nu} \log \overline{1-z_{\nu}}\right) \\
& +2 \pi \sum_{\nu=1}^{n} \operatorname{Re}\left(\overline{c_{\nu}^{\prime}} \log \left(1-z_{\nu}\right)-\overline{c_{\nu}^{\prime \prime}} \log z_{\nu}\right) \\
& +i \pi^{2} \mathbf{c}^{\mathrm{t}} J_{2 n} \overline{\mathbf{c}} .
\end{aligned}
$$

By Lemma 3.1 this gives

$$
\begin{aligned}
\frac{\pi}{2} \operatorname{Re}\left(\sum_{j=1}^{h} \lambda_{j}\right)=\alpha_{0} & +\frac{1}{2} \sum_{\nu=1}^{n} \operatorname{Im}\left(\log z_{\nu} \log \overline{1-z_{\nu}}\right) \\
& -\frac{1}{2} \pi \operatorname{Re}\left(\sum_{\nu=1}^{n}\left(\overline{c_{\nu}^{\prime}} \log \left(1-z_{\nu}\right)-\overline{c_{\nu}^{\prime \prime}} \log z_{\nu}\right)\right)
\end{aligned}
$$

with $\alpha_{0}=-(i / 4) \pi^{2} \mathbf{c}^{\mathrm{t}} J_{2 n} \overline{\mathbf{c}}$, which vanishes if $\mathbf{c}$ is real. Combining this with the elementary formula

$$
\frac{1}{2} \operatorname{Im}(\log z \log \overline{1-z})=\frac{1}{2} \operatorname{Im}(\log z \log (1-z))-\log |z| \arg (1-z)
$$

we get

$$
\begin{aligned}
\frac{\pi}{2} \operatorname{Re}\left(\sum_{j=1}^{h} \lambda_{j}\right)=\alpha_{0} & +\frac{1}{2} \sum_{\nu=1}^{n} \operatorname{Im}\left(\log z_{\nu} \log \left(1-z_{\nu}\right)\right)-\sum_{\nu=1}^{n} \log \left|z_{\nu}\right| \arg \left(1-z_{\nu}\right) \\
& -\frac{1}{2} \pi \operatorname{Re}\left(\sum_{\nu=1}^{n}\left(\overline{c_{\nu}^{\prime}} \log \left(1-z_{\nu}\right)-\overline{c_{\nu}^{\prime \prime}} \log z_{\nu}\right)\right) .
\end{aligned}
$$

The function $\log |z| \arg (1-z)+\operatorname{Im~Li}_{2}(z)$ is called the "Bloch-Wigner dilogarithm." It is the volume of the ideal tetrahedron with parameter $z$ (cf. [NZ], for example). Thus

$$
\operatorname{Vol}\left(M^{\prime}\right)=\sum_{\nu=1}^{n}\left(\log \left|z_{\nu}\right| \arg \left(1-z_{\nu}\right)+\operatorname{Im} \operatorname{Li}_{2}\left(z_{\nu}\right)\right)
$$


Adding (3.4) to (3.5) gives

$$
\begin{aligned}
\operatorname{Vol}\left(M^{\prime}\right)+\frac{\pi}{2} \operatorname{Re}\left(\sum_{j=1}^{h} \lambda_{j}\right)=\alpha_{0}+\frac{1}{2} \sum_{\nu=1}^{n} \operatorname{Im}\left(\log z_{\nu} \log \left(1-z_{\nu}\right)\right)+\sum_{\nu=1}^{n} \operatorname{Im}_{\operatorname{Li}}\left(z_{\nu}\right) \\
\quad-\frac{1}{2} \pi \operatorname{Re}\left(\sum_{\nu=1}^{n}\left(\overline{c_{\nu}^{\prime}} \log \left(1-z_{\nu}\right)-\overline{c_{\nu}^{\prime \prime}} \log z_{\nu}\right)\right) \\
=\alpha_{0}+\sum_{\nu=1}^{n} \operatorname{Im} \mathcal{R}\left(z_{\nu}\right)-\frac{1}{2} \pi \operatorname{Re}\left(\sum_{\nu=1}^{n}\left(\overline{c_{\nu}^{\prime}} \log \left(1-z_{\nu}\right)-\overline{c_{\nu}^{\prime \prime}} \log z_{\nu}\right)\right)
\end{aligned}
$$

which can be written

$$
\begin{aligned}
& \operatorname{Re}\left(\mathcal{V}\left(M^{\prime}\right)+\frac{\pi}{2} \sum_{j=1}^{h} \lambda_{j}\right)= \\
& \quad \alpha_{0}+\operatorname{Re}\left(-i \sum_{\nu=1}^{n}\left(\mathcal{R}\left(z_{\nu}\right)-\frac{i \pi}{2}\left(\overline{c_{\nu}^{\prime}} \log \left(1-z_{\nu}\right)-\overline{c_{\nu}^{\prime \prime}} \log z_{\nu}\right)\right)\right) .
\end{aligned}
$$

This confirms the real part of the formula of Theorem 1. But T. Yoshida shows in [Y1], confirming a conjecture of [NZ], that $\mathcal{V}\left(M^{\prime}\right)+\frac{\pi}{2} \sum_{j=1}^{h} \lambda_{j}$ is a complex analytic function. Since a complex analytic function is determined up to a constant by its real part, the formula of Theorem 1 is proved.

To complete the proof of Theorem 1 we must show that the constant $\alpha$ is in $i \pi^{2} \mathbb{Q}$ if c is rational. We use work of Dupont [D] (that builds on work of Bloch and Wigner and Dupont and Sah), which we now quote.

Let $\mathcal{P}_{\mathbb{C}}$ be the "Bloch group", generated by symbols $\{z\}$ with $z \in \mathbb{C}-\{0,1\}$ subject to the relations

$$
\sum_{i=0}^{4}\left\{\left[a_{0}: \ldots: \widehat{a_{i}}: \ldots: a_{4}\right]\right\}=0 \text { for distinct points } a_{0}, \ldots, a_{4} \in \mathbb{C}-\infty
$$

where $\left[z_{0}: z_{1}: z_{2}: z_{3}\right]$ means the cross-ratio $\left(z_{0}-z_{2}\right)\left(z_{1}-z_{3}\right) /\left(z_{0}-z_{3}\right)\left(z_{1}-z_{2}\right)$.

There is a commutative diagram with exact rows

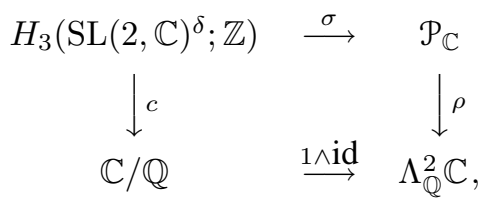

where the map of importance to us is $\rho$, given by the formula

$$
\rho(\{z\})=\frac{\log z}{2 \pi i} \wedge \frac{\log (1-z)}{2 \pi i}+1 \wedge \frac{\mathcal{R}(z)}{2 \pi^{2}} .
$$

Dupont shows that the map $c$ is $2 \widehat{C_{2}}$, where $\widehat{C_{2}}$ is the Cheeger-Chern-Simons class associated to the Chern polynomial $c_{2}$. Our hyperbolic manifold $M^{\prime}$ represents an 
element of $H_{3}\left(\mathrm{SL}(2, \mathbb{C})^{\delta} ; \mathbb{Z}\right)$ and in our terminology his result is that

$$
\sum_{\nu=1}^{n} \rho\left(\left\{z_{\nu}\right\}\right)=1 \wedge 2 \widehat{C_{2}}(M)=1 \wedge \frac{i}{2 \pi^{2}} \mathcal{V}\left(M^{\prime}\right)
$$

This equation will be our starting point.

(Dupont used $\Lambda_{\mathbb{Z}}^{2} \mathbb{C}$ rather than $\Lambda_{\mathbb{Q}}^{2} \mathbb{C}$. They are the same thing: if $s$ and $t$ are integers and $z, w \in \mathbb{C}$ then in $\Lambda_{\mathbb{Z}}^{2} \mathbb{C}$ we have $(s / t) z \wedge w=(s / t) z \wedge t(1 / t) w=s z \wedge(1 / t) w=$ $z \wedge(s / t) w$. For us $\Lambda_{\mathbb{Q}}^{2} \mathbb{C}$ is more convenient.)

Now suppose $M^{\prime}$ results from $M$ by $\left(p_{j}, q_{j}\right)$-Dehn surgery at the $j$-th end. Then, as described at the start of this section,

$$
p_{j} \frac{u_{j}}{2 \pi i}+q_{j} \frac{v_{j}}{2 \pi i}=1
$$

Choose integers $r_{j}$ and $s_{j}$ with $p_{j} s_{j}-q_{j} r_{j}=1$. Then in [NZ] it is shown that

$$
r_{j} \frac{u_{j}}{2 \pi i}+s_{j} \frac{v_{j}}{2 \pi i}=\frac{\lambda_{j}}{2 \pi i}
$$

([NZ] had a different sign due to the differing orientation convention.) Hence, taking wedge product of these two equations,

$$
\frac{u_{j}}{2 \pi i} \wedge \frac{v_{j}}{2 \pi i}=1 \wedge \frac{\lambda_{j}}{2 \pi i}
$$

For $\mathbf{w}=\left(w_{1}, \ldots, w_{2 m}\right)$ and $\mathbf{w}^{\prime}=\left(w_{1}^{\prime}, \ldots, w_{2 m}^{\prime}\right)$ in $\mathbb{C}^{2 m}$ we define $\mathbf{w} \wedge \mathbf{w}^{\prime}=$ $(1 / 2) \sum_{j=1}^{m}\left(w_{j} \wedge w_{m+j}^{\prime}-w_{m+j} \wedge w_{j}^{\prime}\right) \in \Lambda_{\mathbb{Q}}^{2} \mathbb{C}$. It is a formal observation that whenever rational matrices $R$ and $C$ satisfy Lemma 3.2, they will satisfy:

$$
R \mathbf{x}=R \mathbf{y}=0 \quad \Longrightarrow \quad \mathbf{x} \wedge \mathbf{y}=\frac{1}{2} C \mathbf{x} \wedge C \mathbf{y}, \quad \text { for } \mathbf{x}, \mathbf{y} \in \mathbb{C}^{2 n}
$$

( $\mathbb{C}$ could be replaced by any $\mathbb{Q}$-vector space for this.)

Now suppose we have a rational solution $\mathbf{c}$ to $U \mathbf{c}=\mathbf{d}$. We shall denote $Z-\mathbf{c} \pi i$ by $z_{0}$. Using, in turn, (3.8) and (3.7), (3.10), and (3.9), 


$$
\begin{aligned}
1 \wedge \frac{i}{2 \pi^{2}} \mathcal{V}\left(M^{\prime}\right)= & \frac{z}{2 \pi i} \wedge \frac{z}{2 \pi i}+\sum_{\nu=1}^{n} 1 \wedge \frac{\mathcal{R}\left(z_{\nu}\right)}{2 \pi^{2}} \\
= & \frac{z_{0}}{2 \pi i} \wedge \frac{z_{0}}{2 \pi i}+\frac{1}{2}\left(\frac{z}{2 \pi i} \wedge \mathbf{c}+\mathbf{c} \wedge \frac{z}{2 \pi i}\right)-\frac{1}{4} \mathbf{c} \wedge \mathbf{c}+\sum_{\nu=1}^{n} 1 \wedge \frac{\mathcal{R}\left(z_{\nu}\right)}{2 \pi^{2}} \\
= & \frac{1}{2} \sum_{j=1}^{h} \frac{u_{j}}{2 \pi i} \wedge \frac{v_{j}}{2 \pi i}+\frac{1}{2} \sum_{\nu=1}^{n}\left(c_{\nu}^{\prime} \wedge \frac{\log 1-z_{\nu}}{2 \pi i}-c_{\nu}^{\prime \prime} \wedge \frac{\log z_{\nu}}{2 \pi i}\right) \\
& +\sum_{\nu=1}^{n} 1 \wedge \frac{\mathcal{R}\left(z_{\nu}\right)}{2 \pi^{2}} \\
= & \frac{1}{2} \sum_{j=1}^{h} 1 \wedge \frac{\lambda_{j}}{2 \pi i}+\frac{1}{2} \sum_{\nu=1}^{n}\left(1 \wedge c_{\nu}^{\prime} \frac{\log 1-z_{\nu}}{2 \pi i}-1 \wedge c_{\nu}^{\prime \prime} \frac{\log z_{\nu}}{2 \pi i}\right) \\
& +\sum_{\nu=1}^{n} 1 \wedge \frac{\mathcal{R}\left(z_{\nu}\right)}{2 \pi^{2}} .
\end{aligned}
$$

Hence

$$
\begin{aligned}
\frac{i}{2 \pi^{2}} \mathcal{V}\left(M^{\prime}\right)=\frac{1}{2} & \sum_{j=1}^{h} \frac{\lambda_{j}}{2 \pi i}+\frac{1}{2} \sum_{\nu=1}^{n}\left(c_{\nu}^{\prime} \frac{\log 1-z_{\nu}}{2 \pi i}-c_{\nu}^{\prime \prime} \frac{\log z_{\nu}}{2 \pi i}\right) \\
& +\sum_{\nu=1}^{n} \frac{\mathcal{R}\left(z_{\nu}\right)}{2 \pi^{2}}(\bmod \mathbb{Q}),
\end{aligned}
$$

which is the desired rationality result.

Proof of Theorem 2. We assume Theorem 2.4. Suppose we have two c's as in Theorem 2, that is, satisfying the conditions C1, C2, and C3 of Section 2 (cf. Theorem 2.4). Denote their difference by s. Replacing one of these c's by the other in the formula of Theorem 1 changes the formula by $(\pi / 2) \mathbf{s}^{\mathrm{t}} J_{2 n} Z$. To prove Theorem 2 we must show this is a multiple of $i \pi^{2} / 2$. But by Theorem $2.4, \mathrm{~s}^{\mathrm{t}} J_{2 n}$ is a linear combination of the rows of the coefficient matrix $R$ of the consistency condition, so the consistency condition (equation (2.2)) implies that $\mathbf{s}^{\mathrm{t}} J_{2 n} Z$ is $\pi i$ times an integer.

Remark. It is easy to check that the formula for $\mathcal{V}\left(M^{\prime}\right)$ of Theorem 2 is invariant modulo $i \pi^{2} / 6$ under "cocycle moves" to change the triangulation: replace two ideal simplices $\left\langle V_{0}, V_{1}, V_{2}, V_{3}\right\rangle$ and $\left\langle V_{0}^{\prime}, V_{1}, V_{2}, V_{3}\right\rangle$ which have a common face by three$\left\langle V_{0}, V_{0}^{\prime}, V_{1}, V_{2}\right\rangle,\left\langle V_{0}, V_{0}^{\prime}, V_{2}, V_{3}\right\rangle$, and $\left\langle V_{0}, V_{0}^{\prime}, V_{3}, V_{1}\right\rangle$. 


\section{Combinatorics of 3-cycles}

By an $n$-cycle with boundary will be meant any $n$-complex $K$ obtained from a finite disjoint union of closed $n$-simplices by iteratively gluing together pairs of free $(n-$ 1)-faces by simplicial identification maps (a "free" face is one that has not yet been glued) in such a way that any open $q$-simplex still embeds after the identification. If no free faces remain $K$ will be called a closed n-cycle or just an $n$-cycle (these are also called " $n$-dimensional normal pseudo-manifolds" in the literature). Alternatively, 1-cycles are compact quasi-simplicial 1 -manifolds and $n$-cycles can then be defined inductively for $n \geq 2$ as finite quasi-simplicial complexes whose vertex links are connected $(n-$ 1)-cycles. (A "quasi-simplicial complex" is a finite CW-complex built from simplices such that the attaching map $\partial \Delta^{q} \rightarrow K^{q-1}$ for each $q$-simplex is simplicial and is injective on each open $(q-k)$-face of $\Delta^{q}$. The second barycentric subdivision of such a complex is a simplicial complex.) We will not distinguish notationally the complex $K$ and its underlying topological space. The complement $K-K^{n-3}$ of the $(n-3)$-skeleton of an $n$-cycle $K$ is a manifold, and by an orientation of $K$ we mean an orientation of this manifold.

For $n \leq 2$ an $n$-cycle is an $n$-manifold. A 3 -cycle is topologically a manifold except for finitely many singular points where the local structure is that of a cone on a closed connected surface.

To an oriented 3 -simplex $\Delta$ we shall associate a 2 -dimensional bilinear space $J_{\Delta}$ over $\mathbb{Z}$ as follows. As a $\mathbb{Z}$-module $J_{\Delta}$ is generated by the six edges $e_{1}, \ldots, e_{6}$ of $\Delta$ (see Fig. 2) with the relations:

$$
\begin{gathered}
e_{i}-e_{i+3}=0 \text { for } i=1,2,3 . \\
e_{1}+e_{2}+e_{3}=0 .
\end{gathered}
$$

Thus, opposite edges of $\Delta$ represent the same element of $J_{\Delta}$, so $J_{\Delta}$ has three "geometric" generators, and the sum of these three generators is zero. The bilinear form on $J_{\Delta}$ is the non-singular skew-symmetric form given by

$$
\left\langle e_{1}, e_{2}\right\rangle=\left\langle e_{2}, e_{3}\right\rangle=\left\langle e_{3}, e_{1}\right\rangle=-\left\langle e_{2}, e_{1}\right\rangle=-\left\langle e_{3}, e_{2}\right\rangle=-\left\langle e_{1}, e_{3}\right\rangle=1 .
$$

Now suppose $K$ is an oriented 3 -cycle. For each $i$ let $C_{i}$ be the free $\mathbb{Z}$-module on the unoriented $i$-simplices of $K$. Let $J$ be the direct sum $\amalg J_{\Delta}$, summed over the oriented 3 -simplices of $K$. There is a unique reasonable way of defining natural homomorphisms

$$
\alpha: C_{0} \longrightarrow C_{1}
$$

and

$$
\beta: C_{1} \longrightarrow J
$$

Namely, $\alpha$ takes a vertex to the sum of the incident edges (with an edge counted twice if both endpoints are at the given vertex). The $J_{\Delta}$ component of $\beta$ takes an edge $E$ of $K$ to the sum of those edges $e_{i}$ in the edge set $\left\{e_{1}, e_{2}, \ldots, e_{6}\right\}$ of $\Delta$ which are identified with $E$ in $K$. 
The natural basis of $C_{i}$ gives an identification of $C_{i}$ with its dual space and the bilinear form on $J$ gives an identification of $J$ with its dual space. With respect to these identifications, the dual map

$$
\alpha^{*}: C_{1} \longrightarrow C_{0}
$$

is easily seen to map an edge $E$ of $K$ to the sum of its endpoints, and the dual map

$$
\beta^{*}: J \longrightarrow C_{1}
$$

can be described as follows. To each 3 -simplex $\Delta$ of $K$ we have a map $j=j_{\Delta}$ of the edge set $\left\{e_{1}, e_{2}, \ldots, e_{6}\right\}$ of $\Delta$ to the set of edges of $K$ : put $j\left(e_{i}\right)$ equal to the edge that $e_{i}$ is identified with in $K$. For $e_{i}$ in $J_{\Delta}$ we have

$$
\beta^{*}\left(e_{i}\right)=j\left(e_{i+1}\right)-j\left(e_{i+2}\right)+j\left(e_{i+4}\right)-j\left(e_{i+5}\right) \quad \text { (indices mod 6). }
$$

This is shown pictorially in Fig. 4.

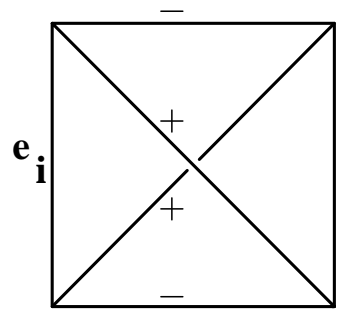

Figure 4

We shall show that $\operatorname{Im} \beta \subseteq \operatorname{Ker} \beta^{*}$. Since $\operatorname{Ker} \beta^{*}=(\operatorname{Im} \beta)^{\perp}$, the form on $J$ then induces a form on $\operatorname{Ker} \beta^{*} / \operatorname{Im} \beta$ which is non-degenerate on $\left(\operatorname{Ker} \beta^{*} / \operatorname{Im} \beta\right) /\{$ Torsion $\}$. We shall denote this form also by $\langle$,$\rangle .$

Let $K_{0}$ be the result of removing a small open cone neighborhood of each vertex $V$ of $K$, so $\partial K_{0}$ is the disjoint union of the links $L_{V}$ of the vertices of $K$.

Theorem 4.1. The sequence

$$
\text { J: } \quad 0 \longrightarrow C_{0} \stackrel{\alpha}{\longrightarrow} C_{1} \stackrel{\beta}{\longrightarrow} J \stackrel{\beta^{*}}{\longrightarrow} C_{1} \stackrel{\alpha^{*}}{\longrightarrow} C_{0} \longrightarrow 0
$$

is a chain complex. Tensored with $\mathbb{Z}\left[\frac{1}{2}\right]$, it is exact except in the middle, where its homology is the first homology of $\partial K_{0}$ :

$$
\left(\operatorname{Ker} \beta^{*} / \operatorname{Im} \beta\right) \otimes \mathbb{Z}\left[\frac{1}{2}\right]=H_{1}\left(\partial K_{0} ; \mathbb{Z}\left[\frac{1}{2}\right]\right)=\coprod_{V \in K^{0}} H_{1}\left(L_{V} ; \mathbb{Z}\left[\frac{1}{2}\right]\right)
$$

Moreover, the bilinear form $\langle$,$\rangle on \left(\operatorname{Ker} \beta^{*} / \operatorname{Im} \beta\right) \otimes \mathbb{Z}\left[\frac{1}{2}\right]$ is twice the intersection form on $H_{1}\left(\partial K_{0} ; \mathbb{Z}\left[\frac{1}{2}\right]\right)$.

Note that only vertices at which $K$ is not a manifold contribute their homology in this theorem. Thus the chain complex $\mathcal{J}$ computes the "local homology" of the singularities of $K$. 
Remark. In [NZ, Theorem 2.2 to Proposition 2.5] combinatorial results equivalent to the above Theorem were proved under the extra assumption that the complement of the vertices, $K-K^{0}$, carries a complete hyperbolic structure of finite volume.

We will need the homology of $\mathcal{J}$ without tensoring with $\mathbb{Z}\left[\frac{1}{2}\right]$, but the result is more technical. We number homology groups of $\mathcal{J}$ as follows:

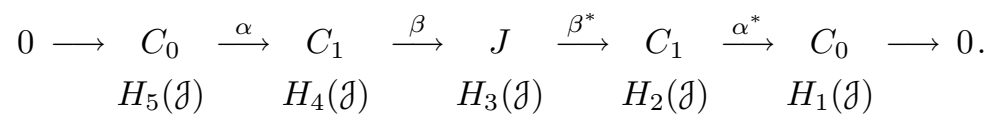

Theorem 4.2. The homology groups $H_{i}(\mathcal{J})$ are

$$
\begin{gathered}
H_{5}(\mathcal{J})=0, \quad H_{4}(\mathcal{J})=\mathbb{Z} / 2, \quad H_{1}(\mathcal{J})=\mathbb{Z} / 2, \\
H_{3}(\mathcal{J})=\mathcal{H} \oplus H^{1}(K ; \mathbb{Z} / 2), \quad H_{2}(\mathcal{J})=H_{1}(K ; \mathbb{Z} / 2),
\end{gathered}
$$

where $\mathcal{H}=\operatorname{Ker}\left(H_{1}\left(\partial K_{0} ; \mathbb{Z}\right) \rightarrow H_{1}\left(K_{0} ; \mathbb{Z} / 2\right)\right)$. The isomorphism $H_{3}(\mathcal{J}) /\{$ Torsion $\}$ $\rightarrow \mathcal{H}$ is an isometry for the form on $H_{3}(\mathcal{J})=\operatorname{Ker} \beta^{*} / \operatorname{Im} \beta$ and the intersection form on $\mathcal{H} \subseteq H_{1}\left(\partial K_{0}\right)=\coprod H_{1}\left(L_{V}\right)$.

Remark. The isomorphism of $H_{2}(\mathcal{J})$ with $H_{1}(K ; \mathbb{Z} / 2)$ is given by the obvious mapan element of Ker $\alpha^{*}$ represents a modulo-2 simplicial 1-cycle in $K$. The torsion of $H_{3}(\mathcal{J})$ then follows by the universal coefficient theorem. The map of $H_{3}(\mathcal{J}) /\{$ Torsion\} to $\mathcal{H}$ is less obvious, and is pictured in Fig. 9 below. In Theorem 5.1 we give a more direct computation of $H_{3}(\mathcal{J})$ in terms of cohomology, which we need later, but its proof uses the above theorem.

Proof. We shall work over $\mathbb{Z}$. The version over $\mathbb{Z}\left[\frac{1}{2}\right]$ follows by the same proof, or directly from the result over $\mathbb{Z}$.

To show that $\mathcal{J}$ is a chain complex we must show $\beta \circ \alpha=0, \beta^{*} \circ \beta=0$, and $\alpha^{*} \circ \beta^{*}=0$. The first and third of these equations are dual to each other and hence equivalent, and the third equation is clear from Fig. 4. For the second equation note that, for an edge $E$ of $K, \beta^{*} \beta(E)$ is a sum of contributions $\pm E^{\prime}$ over edges $E^{\prime}$ which are adjacent to $E$ in some 3 -simplex $\Delta$ of $K$. Each such $E^{\prime}$ spans with $E$ a 2 -simplex face of $\Delta$ and contributes also with opposite sign to $\beta^{*} \beta(E)$ for the 3 -simplex on the other side of this face. Thus $\beta^{*} \beta(E)$ is zero.

We must next discuss the homology groups $H_{i}(\mathcal{J})$.

$H_{1}(\mathcal{J})$ is $\operatorname{Coker}\left(\alpha^{*}\right)$ and we must show it is $\mathbb{Z} / 2$. If $\epsilon: C_{0} \rightarrow \mathbb{Z} / 2$ is the map which takes an element to the mod- 2 sum of its coefficients, then certainly $\operatorname{Im}\left(\alpha^{*}\right) \subseteq \operatorname{Ker}(\epsilon)$. For any 2-simplex $F$ of $K$, let $E_{1}, E_{2}$, and $E_{3}$ be the three edges of $F$ (which may not be distinct, since $K$ is just quasi-simplicial). Then $\alpha^{*}\left(E_{1}+E_{2}-E_{3}\right)=2 V$, where $V$ is the common vertex of $E_{1}$ and $E_{2}$ in $F$, and $\alpha^{*}\left(E_{2}-E_{3}\right)=V-V^{\prime}$, where $V^{\prime}$ is the vertex at the other end of $E_{1}$. Since $\operatorname{Ker}(\epsilon)$ is generated by elements of these two types, $\operatorname{Im}\left(\alpha^{*}\right)=\operatorname{Ker}(\epsilon)$, as was to be proved.

Any element of $\operatorname{Ker}\left(\alpha^{*}\right)$, taken modulo 2 , is a simplicial mod- 21 -cycle in $K$. Thus we have a map $\operatorname{Ker}\left(\alpha^{*}\right) \rightarrow H_{1}(K ; \mathbb{Z} / 2)$. This map vanishes on $\operatorname{Im}\left(\beta^{*}\right)$ (see Fig. 4), so it induces a map $H_{2}(\mathcal{J}) \rightarrow H_{1}(K ; \mathbb{Z} / 2)$. We claim this is an isomorphism. 
A closed simplicial path in $K$ represents an element of $H_{1}(K ; \mathbb{Z} / 2)$ (the mod- 2 sum of the 1 -simplices along the path) and $H_{1}(K ; \mathbb{Z} / 2)$ is generated by such elements. If the path has odd length, it can be modified to have even length by replacing some 1-simplex $E$ by the sum of the other two edges of some 2-simplex containing $E$. Once it has even length, the alternating sum of the 1-simplices along the path is an element of $\operatorname{Ker}\left(\alpha^{*}\right)$ which represents the given element of $H_{1}(K ; \mathbb{Z} / 2)$. In particular, the map $H_{2}(\mathcal{J}) \rightarrow H_{1}(K ; \mathbb{Z} / 2)$ is onto.

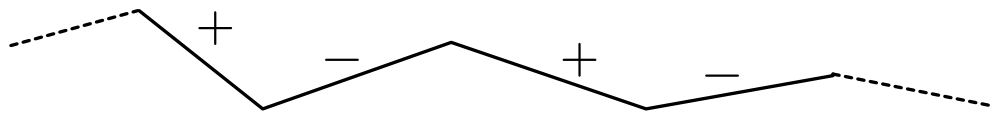

Figure 5

$H_{2}(\mathcal{J})$ is generated by such alternating sums along paths, so we can draw elements of $H_{2}(\mathcal{J})$ as in Fig. 5. Alternating paths as in Fig. 4 give the relations. Now if $F$ and $F^{\prime}$ are 2-simplices with a common edge $E$, and $E_{1}$ and $E_{2}$ (respectively $E_{1}^{\prime}$ and $E_{2}^{\prime}$ ) are the other two edges of $F$ (respectively $F^{\prime}$ ), then any occurrence of $E_{1}-E_{2}$ in an alternating path can be replaced by $E_{1}^{\prime}-E_{2}^{\prime}$ without changing the represented element of $H_{2}(\mathcal{J})$ (see Fig. 6a; there is an orientation of $E$ determined by $E_{1}-E_{2}$ which should agree with the one determined by $E_{1}^{\prime}-E_{2}^{\prime}$ ). Indeed, if $F$ and $F^{\prime}$ represent adjacent vertices in the link of $E$ then this uses a single application of a relation of the type in Fig. 4, so in general it follows from the connectedness of the link of $E$. We therefore denote $E_{1}-E_{2}$ by $E^{+-}$or $E^{-+}$(this assumes an implicit orientation of $E$ ) and draw it as in Fig. 6b.
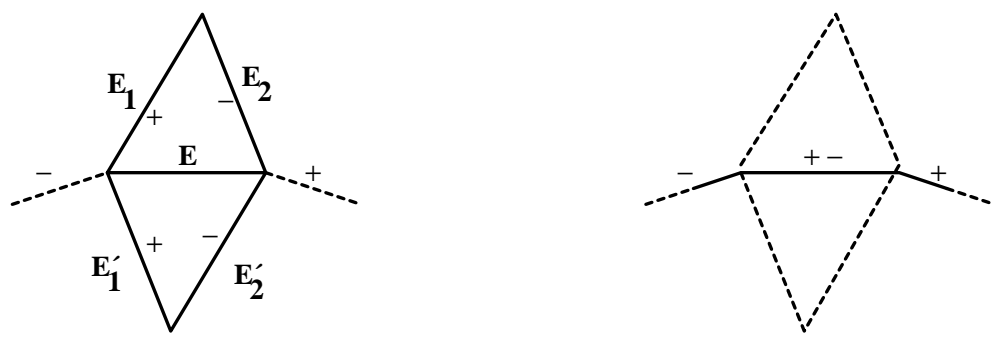

Figure 6 (a) and (b)

In a similar way, the connectedness of the link at a vertex easily implies that the configuration of Fig. 7a represents 0 in $H_{2}(\mathcal{J})$, so an $E$ followed by a $-E^{\prime}$ is equivalent to $E^{+-}$followed by $E^{\prime+-}$ (Fig. 7b).

Thus any alternating path is equivalent to the corresponding path of ${ }^{+-}$edges, or, doing the conversion in the opposite direction around the path, also to the corresponding path of -+ edges. In particular, it is equivalent to its own negative, so the signs on the path are irrelevant to the represented element in $H_{2}(\mathcal{J})$. Moreover, the boundary of a 2-simplex represents 0 (since $E_{1}^{+-}+E_{2}^{+-}+E_{3}^{+-} \sim E_{1}^{+-}+E_{2}-E_{3}=0$ ), so $H_{2}(\mathcal{J})$ is $H_{1}(K ; \mathbb{Z} / 2)$, as claimed.

The computation of $H_{4}(\mathcal{J})$ and $H_{5}(\mathcal{J})$, as well as the torsion in $H_{3}(\mathcal{J})$, now follows by standard duality arguments (the universal coefficient theorem for cohomology), since 


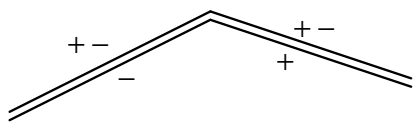

(a)

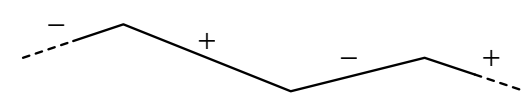

$\iint$

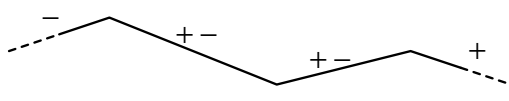

(b)

Figure 7

our chain complex is self-dual. It remains to compute the free part of $H_{3}(\mathcal{J})$. To do so we will need to define maps $\gamma: H_{3}(\mathcal{J}) \rightarrow \mathcal{H}$ and $\delta: H_{1}\left(\partial K_{0}\right) \rightarrow H_{3}(\mathcal{J})$.

Recall that $K_{0}$ denotes the result of removing a small open cone neighborhood of each vertex in $K . K_{0}$ can be constructed by gluing truncated tetrahedra (Fig. 8).

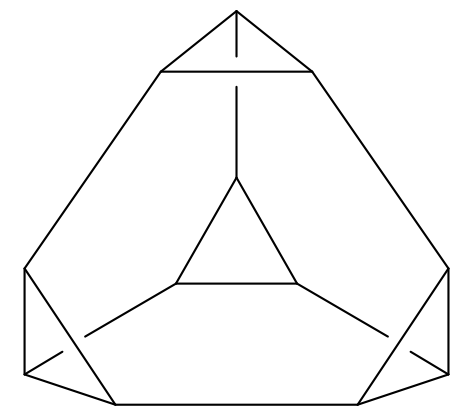

Figure 8

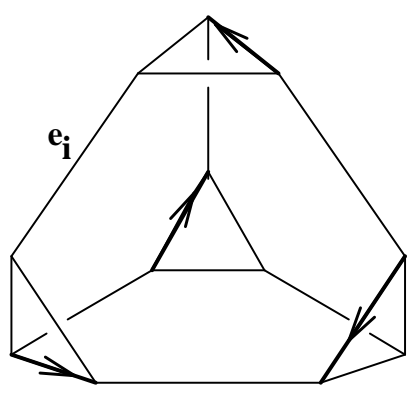

Figure 9

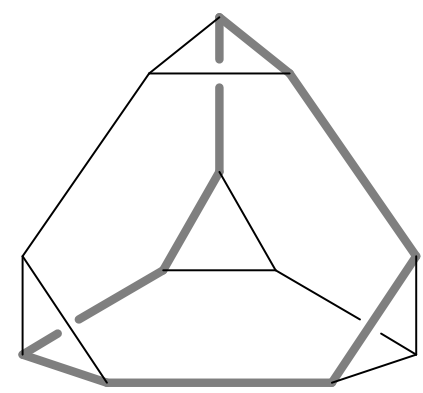

Figure 10

Definition of $\gamma: H_{3}(\mathcal{J}) \rightarrow \mathcal{H}$. The triangular faces of the truncated tetrahedra give a quasi-simplicial triangulation of $\partial K_{0}$. Let $S_{i}\left(\partial K_{0}\right), Z_{i}\left(\partial K_{0}\right)$, and $B_{i}\left(\partial K_{0}\right)$ be the groups of simplicial chains, cycles, and boundaries for this triangulation. Let $\Delta$ be a 3-simplex of $K$. To an edge $e_{i}$ of this simplex we associate a simplicial 1-chain $\gamma_{0}\left(e_{i}\right) \in S_{1}\left(\partial K_{0}\right)$ as in Fig. 9. For opposite edges $e_{i}$ and $e_{i+3}(1 \leq i \leq 3)$ of $\Delta$ one 
has $\gamma_{0}\left(e_{i}\right)=\gamma_{0}\left(e_{i+3}\right)$. Also, $\gamma_{0}\left(e_{1}+e_{2}+e_{3}\right)$ is a boundary cycle. Thus $\gamma_{0}$ induces a map $\gamma_{1}: J \rightarrow S_{1}\left(\partial K_{0}\right) / B_{1}\left(\partial K_{0}\right)$. Moreover, it is easy to see that $\gamma_{1}$ maps $\operatorname{Im} \beta$ to boundaries and maps $\operatorname{Ker} \beta^{*}$ to cycles, so it induces a map

$$
\gamma: H_{3}(\mathcal{J})=\operatorname{Ker} \beta^{*} / \operatorname{Im} \beta \rightarrow H_{1}\left(\partial K_{0}\right)
$$

Note that, if we work modulo 2 , then $\gamma_{0}\left(e_{i}\right)$ differs from the geometric representative for $\beta^{*}\left(e_{i}\right)$ by a 1 -boundary in $\Delta$ (Fig. 10). Thus $\gamma$ followed by the map $H_{1}\left(\partial K_{0}\right) \rightarrow$ $H_{1}\left(K_{0} ; \mathbb{Z} / 2\right)$ is zero; that is

$$
\operatorname{Im} \gamma \subseteq \mathcal{H}=\operatorname{Ker}\left(H_{1}\left(\partial K_{0}\right) \rightarrow H_{1}\left(K_{0} ; \mathbb{Z} / 2\right)\right)
$$

Definition of $\delta: H_{1}\left(\partial K_{0}\right) \longrightarrow H_{3}(\mathcal{\partial})$. We use the dual cell decomposition of the quasitriangulation of $\partial K_{0}$ to compute $H_{1}\left(\partial K_{0}\right)$. A simple cellular path $C$ in the dual cell decomposition of $\partial K_{0}$ determines a vertex of each 2-simplex it passes through - the vertex common to the two edges of the 2 -simplex that it crosses - and a sign + or according as $C$ goes counterclockwise or clockwise around this vertex. A vertex of a 2-simplex of $\partial K_{0}$ corresponds to an edge of a 3-simplex $\Delta$ of $K$, and hence to an element $e$ of $J=\coprod J_{\Delta}$. Define $\delta(C)$ to be the signed sum of these elements $e$ over all 2-simplices of $\partial K_{0}$ that $C$ crosses (Fig. 11). By inspection, $\beta^{*} \delta(C)=0$, so $\delta(C) \in \operatorname{Ker} \beta^{*}$. We consider $\delta(C)$ as an element of $\operatorname{Ker} \beta^{*} / \operatorname{Im} \beta=H_{3}(\mathcal{J})$.

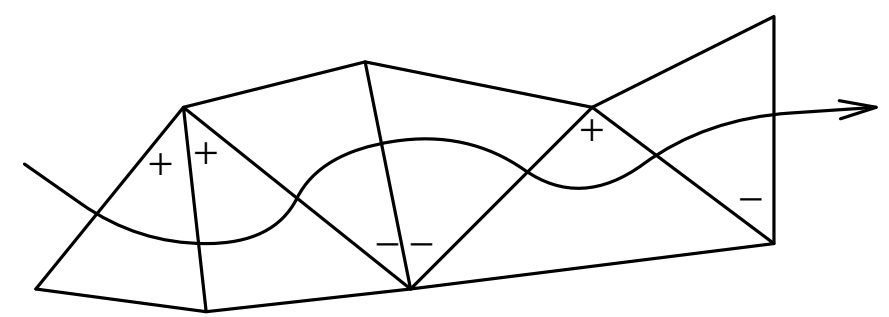

Figure 11

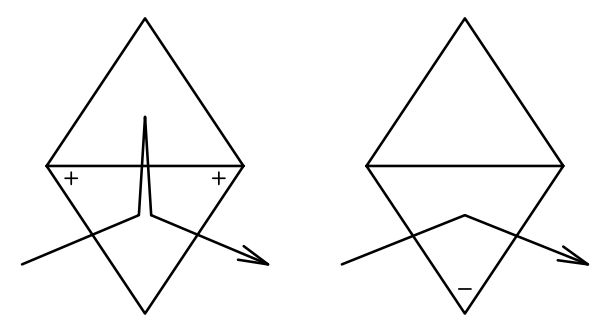

Figure 12

The definition of $\delta$ extends in the obvious fashion to arbitrary closed paths in the dual cell complex of $\partial K_{0}$ (the contribution where a path back-tracks, i.e., enters a 2-simplex and immediately departs across the same edge is zero). Eliminating back-tracking in a closed path $C$ does not change the value of $\delta(C)$, see Fig. 12. If the path $C$ is the boundary path of a 2-cell, then $\delta(C)$ is $\beta$ of the corresponding edge of $K$, so it is zero 
in $H_{3}(\mathcal{J})$. It follows that $\delta(C) \in H_{3}(\mathcal{J})$ only depends on the homology class of $C$, so $\delta$ gives a well-defined homomorphism from $H_{1}\left(\partial K_{0}\right)$ to $H_{3}(\mathcal{J})$.

\section{Lemma 4.3.}

(i) $\gamma \circ \delta: H_{1}\left(\partial K_{0}\right) \rightarrow H_{1}\left(\partial K_{0}\right)$ is multiplication by 2.

(ii) $\gamma$ and $\delta$ are isomorphisms after tensoring with $\mathbb{Z}\left[\frac{1}{2}\right]$.

(iii) $\langle\delta x, \delta y\rangle=2 x \cdot y$, where $x \cdot y$ means intersection form in $H_{1}\left(\partial K_{0}\right)$.

(iv) $\gamma a \cdot \gamma b=2\langle a, b\rangle$ for $a, b \in H_{3}(\mathcal{J})$.

(v) $x \cdot \gamma a=\langle\delta x, a\rangle$ for $a \in H_{3}(\mathcal{J})$ and $x \in H_{1}\left(\partial K_{0}\right)$, that is, $\gamma: H_{3}(\mathcal{J}) /\{$ Torsion $\}$ $\rightarrow H_{1}\left(\partial K_{0}\right)$ and $\delta: H_{1}\left(\partial K_{0}\right) \rightarrow H_{3}(\mathcal{\partial}) /\{$ Torsion $\}$ are adjoint maps with respect to the forms on $H_{1}\left(\partial K_{0}\right)$ and $H_{3}(\mathcal{J}) /\{$ Torsion $\}$.

Proof. For (i) it suffices to show that $\gamma \delta(C)$ is homologous to $2 C$ for a simple closed cellular path $C$. Now (see Fig. 13), $\gamma \delta(C)$ will consist of contributions "near" $C$ and contributions "far from" $C$.

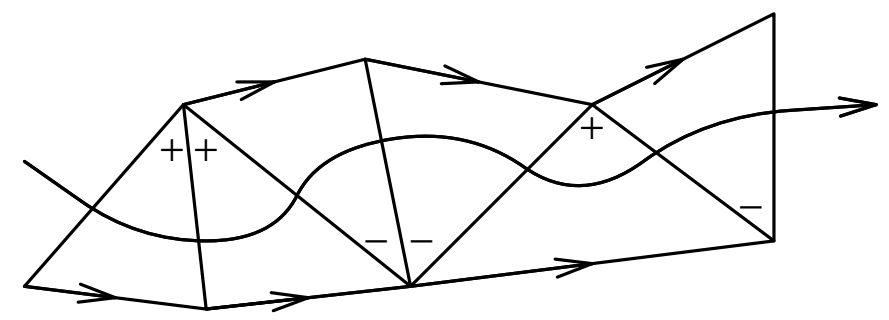

Figure 13a. The "near" contributions.

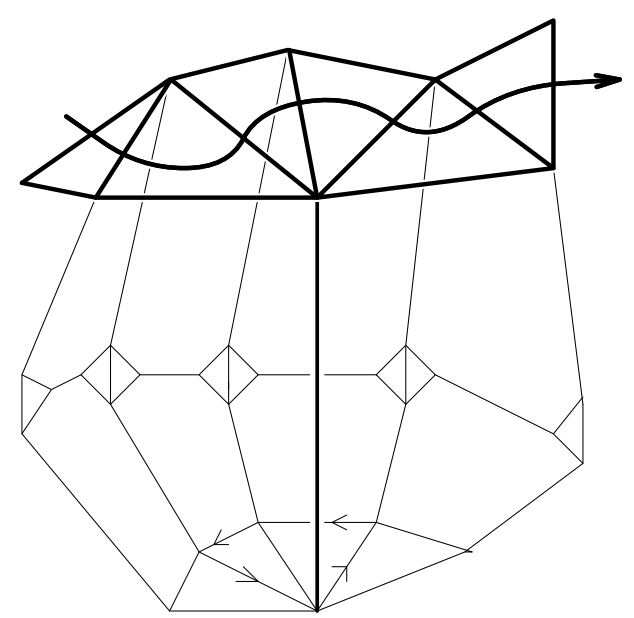

Figure 13b. The "far" contributions.

Contributions of the various types may overlap, but this does not affect the argument. By inspection one sees that the "near" contribution is homologous to $2 C$ (it is the boundary of a regular neighborhood of $C$ ), while each "far" contribution is located at the far end of an edge of $K$ which starts at a vertex of a 2-simplex of $\partial K_{0}$ through which 
$C$ passes, and is a null-homologous loop of length equal to the number of 2-simplices of $\partial K_{0}$ that $C$ passes through as it passes that vertex. Thus (i) holds.

Since we already know that $H_{3}(\mathcal{J})$ has only even torsion, to deduce (ii) from (i) it suffices to show that the ranks of $H_{3}(\mathcal{J})$ and $H_{1}\left(\partial K_{0}\right)$ agree. But the rank of $H_{3}(\mathcal{J})$ equals the Euler characteristic of our chain complex, which is $n_{0}-n_{1}+2 n_{3}-n_{1}+n_{0}=$ $2\left(n_{0}-n_{1}+n_{3}\right)$, where $n_{i}$ is the number of $i$-simplices of $K$. Since $2 n_{3}=n_{2}$ (every 2-simplex is on two 3-simplices and every 3 -simplex has four 2-faces), $2\left(n_{0}-n_{1}+n_{3}\right)=$ $2\left(n_{0}-n_{1}+n_{2}-n_{3}\right)=2 \chi(K)$. The desired equation $2 \chi(K)=\operatorname{rank} H_{1}\left(\partial K_{0}\right)$ now follows from the fact that the Euler characteristic of a compact 3-manifold is half the Euler characteristic of its boundary.

Note that (iii) through (v) are mutually equivalent, given (i) and (ii). (iii) is proved in [NZ, Section 3] (in [NZ] $\partial K_{0}$ consists of tori, but this is not used in the proof) but for completeness we give a proof here. Let $S_{1}$ and $S_{1}^{\prime}$ be the groups of simplicial 1-chains of $\partial K_{0}$ and 1-chains for the dual cell complex of $\partial K_{0}$. Intersection number defines a bilinear form $S_{1}^{\prime} \times S_{1} \rightarrow \mathbb{Z}$, which induces the usual intersection form on $H_{1}\left(\partial K_{0}\right)$. By inspection one sees that, for a closed cellular path $C$ in the dual cell complex of $\partial K_{0}$ and an edge $e$ of a 3-simplex of $K, C \cdot \gamma_{0}(e)=\langle\delta(C), e\rangle$ (on the right of this equation $e$ is interpreted as an element of $J$ ). Part (v) follows, and (iii) and (iv) follow from (v) - for instance $x \cdot(2 y)=x \cdot \gamma \delta(y)=\langle\delta(x), \delta(y)\rangle$.

The proof of the theorem is now easily completed. A standard duality argument shows that the kernel $K$ of $H_{1}\left(\partial K_{0}\right) \rightarrow H_{1}\left(K_{0}\right)$ satisfies $K=K^{\perp}$ (orthogonal complement with respect to the intersection form) and is hence a direct summand of $H_{1}\left(\partial K_{0}\right)$ of rank $h=\left(\operatorname{rank} H_{1}\left(\partial K_{0}\right)\right) / 2$. It follows that $\mathcal{H}=\operatorname{Ker}\left(H_{1}\left(\partial K_{0}\right) \rightarrow H_{1}(K ; \mathbb{Z} / 2)\right)$ has index $2^{h}$ in $H_{1}\left(\partial K_{0}\right)$. On the other hand, since the intersection form on $H_{1}\left(\partial K_{0}\right)$ and the form $\langle$,$\rangle on H_{3}(\mathcal{J}) /\{$ Torsion $\}$ are both non-singular, part (iv) of the lemma implies that $\operatorname{Im} \gamma$ has index $2^{h}$ in $H_{1}\left(\partial K_{0}\right)$. Since $\mathcal{H}$ contains $\operatorname{Im} \gamma$, they are equal.

Remark and Definition. $\delta(C)$ can be defined as above for any closed path $C$ in $\partial K_{0}$ which is in general position with respect to the 1-skeleton of the quasi-triangulation of $\partial K_{0}$. In the next section we will need an analogous construction for closed curves $C$ in $K$ which are in general position with respect to the 2-skeleton of $K$. We shall therefore simply say that a path in $\partial K_{0}$ or in $K$ is general if it is in general position with respect to the 1-skeleton of $\partial K_{0}$, respectively the 2-skeleton of $K$. Let $C$ be such a closed path in $\partial K_{0}$. We say $C$ has no back-tracking if it never enters a 2-simplex and immediately leaves again across the same edge. Similarly, we say a closed path in $K$ has no backtracking if it never enters a 3-simplex of $K$ and immediately leaves it again across the same face.

\section{Cohomological computation of $H_{3}(\mathcal{J})$}

The main result of this section is Theorem 5.1, which gives an explicit computation of $H_{3}(\mathcal{J})$ in terms of two maps $\gamma^{\prime}: H_{3}(\mathcal{J}) \rightarrow H^{1}\left(\partial K_{0} ; \mathbb{Z}\right)$ and $\gamma_{2}^{\prime}: H_{3}(\mathcal{J} \otimes \mathbb{Z} / 2) \rightarrow$ $H^{1}\left(K_{0} ; \mathbb{Z} / 2\right)$. We must first define these maps.

$\gamma^{\prime}: H_{3}(\mathcal{J}) \rightarrow H^{1}\left(\partial K_{0} ; \mathbb{Z}\right)$ is just the Poincare dual of the map $\gamma$ of the previous section, that is $\gamma^{\prime}=P D \circ \gamma$, where $P D: H_{1}\left(\partial K_{0}\right) \rightarrow H^{1}\left(\partial K_{0}\right)$ is the Poincaré duality 
isomorphism. We can also express it as the dual of the map $\delta: H_{1}\left(\partial K_{0}\right) \rightarrow H_{3}(\mathcal{J})$ as follows:

$$
\gamma^{\prime}(a)(c)=\langle a, \delta(c)\rangle \quad \text { for } c \in H_{1}\left(\partial K_{0}\right) .
$$

Indeed, $\gamma^{\prime}(a)(c)=P D(\gamma(a))(c)=\gamma(a) \cdot c=\langle a, \delta(c)\rangle$, where the last equality is by Lemma 4.3.

In a similar way we shall describe $\gamma_{2}^{\prime}: H_{3}(\mathcal{J} \otimes \mathbb{Z} / 2) \rightarrow H^{1}\left(K_{0} ; \mathbb{Z} / 2\right)$ as the dual of a map $\delta_{2}: H_{1}\left(K_{0} ; \mathbb{Z} / 2\right) \rightarrow H_{3}(\mathcal{J} \otimes \mathbb{Z} / 2):$

$$
\gamma_{2}^{\prime}(a)(c)=\left\langle a, \delta_{2}(c)\right\rangle \quad \text { for } c \in H_{1}\left(K_{0} ; \mathbb{Z} / 2\right),
$$

where $\delta_{2}$ is defined as follows.

If $c \in H_{1}\left(K_{0} ; \mathbb{Z} / 2\right)$ is represented by a general closed path $C$ in $K_{0}$, then each time $C$ passes through a 3 -simplex $\Delta$ from one face to another, it determines an edge $e$ of $\Delta$, namely the edge common to the two faces, and hence an element, also denoted $e$, of $J \otimes \mathbb{Z} / 2$. We let $\delta_{2}(c)$ be the class in $H_{3}(\mathcal{J} \otimes \mathbb{Z} / 2)$ of the sum of these elements $e$. The proof that this sum is indeed a cycle, i.e., in $\operatorname{Ker}\left(\beta^{*} \otimes \mathbb{Z} / 2\right)$, and that modulo $\operatorname{Im}(\beta \otimes \mathbb{Z} / 2)$ it only depends on the homology class of $C$, is entirely analogous to the corresponding proof for $\delta$.

Let $\iota: H_{3}(\mathcal{J}) \rightarrow H_{3}(\mathcal{J} \otimes \mathbb{Z} / 2)$ be the natural map. We shall be interested in the composition

$$
\gamma_{2}^{\prime} \iota: H_{3}(\mathcal{J}) \stackrel{\iota}{\rightarrow} H_{3}(\mathcal{J} \otimes \mathbb{Z} / 2) \stackrel{\gamma_{2}^{\prime}}{\rightarrow} H^{1}\left(K_{0} ; \mathbb{Z} / 2\right) .
$$

Theorem 5.1. The following diagram is a pullback diagram:

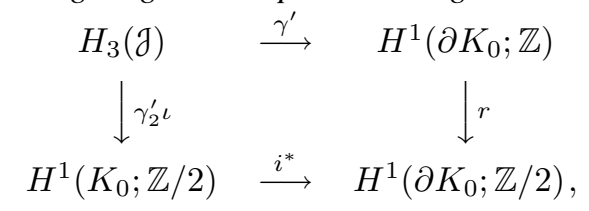

where $i^{*}$ is induced by $i: \partial K_{0} \rightarrow K_{0}$ and $r$ is reduction modulo 2. Equivalently, the following sequence is exact:

$$
0 \rightarrow H_{3}(\mathcal{J}) \stackrel{\left(\gamma^{\prime}, \gamma_{2}^{\prime} \iota\right)}{\longrightarrow} H^{1}\left(\partial K_{0} ; \mathbb{Z}\right) \oplus H^{1}\left(K_{0} ; \mathbb{Z} / 2\right) \stackrel{r-i^{*}}{\longrightarrow} H^{1}\left(\partial K_{0} ; \mathbb{Z} / 2\right) \rightarrow 0 .
$$

Proof. The commutativity of the diagram is immediate from (5.1) and (5.2) and the fact that if $C$ is a cellular curve on $\partial K_{0}$ and $C_{2}$ is the result of pushing $C$ inside $K_{0}$, then $\delta_{2}\left(C_{2}\right)=\delta(C)(\bmod 2)$.

The rest of the proof will take several steps.

Step 1. $\gamma_{2}^{\prime}: H_{3}(\mathcal{J} \otimes \mathbb{Z} / 2) \rightarrow H^{1}\left(K_{0} ; \mathbb{Z} / 2\right)$ is surjective.

Until further notice coefficient group $\mathbb{Z} / 2$ is understood.

Step 1 is most easily seen by translating to homology via the Poincaré duality isomorphism $H^{1}\left(K_{0}\right) \rightarrow H_{2}\left(K_{0}, \partial K_{0}\right)$. Note that $H_{2}\left(K_{0}, \partial K_{0}\right)=H_{2}(K)$ by the exact sequence for the pair $\left(K, K-K_{0}\right)$ and excision. We shall construct a map $\gamma_{2}: H_{3}(\mathcal{J} \otimes$ $\mathbb{Z} / 2) \rightarrow H_{2}(K)$ and show it is the Poincaré dual of $\gamma_{2}^{\prime}: H_{3}(\mathcal{J} \otimes \mathbb{Z} / 2) \rightarrow H^{1}\left(K_{0}\right)$.

Let, for the moment, $S_{2}, Z_{2}$, and $B_{2}$ denote the groups of simplicial 2-chains, 2 -cycles, and 2-boundaries of $K$ with coefficients $\mathbb{Z} / 2$. For an edge $e$ of a 3 -simplex 
$\Delta$ of $K$, let $\gamma_{2}(e) \in S_{2}$ be the sum of the two 2-simplices represented by the two faces of $\Delta$ that meet in $e$. This clearly induces a map $\gamma_{2}: J \rightarrow S_{2} / B_{2}$. Moreover, it is geometrically clear that this map maps $\operatorname{Ker}\left(\beta^{*} \otimes \mathbb{Z} / 2\right)$ to cycles and $\operatorname{Im}(\beta \otimes \mathbb{Z} / 2)$ to zero, and hence induces a map

$$
\gamma_{2}: H_{3}(\mathcal{J} \otimes \mathbb{Z} / 2) \rightarrow H_{2}(K) .
$$

Now for an edge $e$ of a 3-simplex of $k$ and a closed curve in $K_{0}$, we have by inspection, $\gamma_{2}(e) \cdot C=\left\langle e, \delta_{2}(C)\right\rangle(\bmod 2)$. Thus, for $a \in J$ we have $\gamma_{2}(a) \cdot C=\left\langle a, \delta_{2}(C)\right\rangle=$ $\gamma_{2}^{\prime}(a)(C)$, whence $\gamma_{2}^{\prime}$ is indeed the Poincaré dual of $\gamma_{2}$.

Now note that any modulo-2 simplicial 2-cycle $Z \in Z_{2}$ is a sum of an even number of 2-simplices, since $\partial Z=0$ and the boundary of a single 2-simplex is the sum of an odd number of 1-simplices. Call two 2-simplices of $K$ "adjacent" if they lie on a common 3 -simplex and call their sum an "adjacent pair". Any sum of two 2-simplices can be rewritten modulo 2 as a sum of adjacent pairs (connect the two 2 -simplices by a path in $K$ and form adjacent pairs from the successive 2-simplices that the path meets). Thus any $Z \in Z_{2}$ is a sum of adjacent pairs. Each of these adjacent pairs determines an edge of a 3-simplex, hence an element $e$ of $J \otimes \mathbb{Z} / 2$. Let $a$ be the sum of these elements $e$. Clearly $Z=\gamma_{2}(a)$, and the fact that $\partial Z=0$ translates directly to $a \in \operatorname{Ker}\left(\beta^{*} \otimes \mathbb{Z} / 2\right)$. Thus $\gamma_{2}: H_{3}(\mathcal{J} \otimes \mathbb{Z} / 2) \rightarrow H_{2}(K)$ is surjective. Hence $\gamma_{2}^{\prime}$ is surjective.

Step 2. The following sequence is exact (coefficients $\mathbb{Z} / 2$ ):

$$
0 \rightarrow H_{1}\left(K_{0}\right) \stackrel{\delta_{2}}{\longrightarrow} H_{3}(\mathcal{J} \otimes \mathbb{Z} / 2) \stackrel{\gamma_{2}^{\prime}}{\longrightarrow} H^{1}\left(K_{0}\right) \rightarrow 0 .
$$

We have just shown the surjectivity of $\gamma_{2}^{\prime}$ and the injectivity of $\delta_{2}$ follows from this and equation (5.2), since $\delta_{2}(c)=0$ implies $\gamma_{2}^{\prime}(a)(c)=0$ for all $a$, hence $x(c)=0$ for all $x \in H^{1}\left(K_{0}\right)$, hence $c=0$.

The equation $\gamma_{2}^{\prime} \delta_{2}=0$ is equivalent, by the definition of $\gamma_{2}^{\prime}$, to the condition that $\left\langle\delta_{2}(C), \delta_{2}\left(C^{\prime}\right)\right\rangle=0(\bmod 2)$ for any two closed curves $C$ and $C^{\prime}$ in $K_{0}$. We can assume each curve is general, and then it is easy to see that $\left\langle\delta_{2}(C), \delta_{2}\left(C^{\prime}\right)\right\rangle(\bmod 2)$ counts the number of instances of $C$ and $C^{\prime}$ passing through the same 2-face of a 3simplex of $K$. But for each such instance, they pass through the same face viewed as a face of the adjacent 3-simplex, so the total number is zero modulo 2 .

We now know $\operatorname{Im} \delta_{2} \subseteq \operatorname{Ker} \gamma_{2}^{\prime}$, and to show this inclusion is an equality it suffices to show that $\operatorname{dim} H_{3}(\mathcal{J} \otimes \mathbb{Z} / 2)=\operatorname{dim} H_{1}\left(K_{0}\right)+\operatorname{dim} H^{1}\left(K_{0}\right)$, that is, $\operatorname{dim} H_{3}(\mathcal{J} \otimes \mathbb{Z} / 2)=$ $2 \operatorname{dim} H_{1}\left(K_{0}\right)$. But Theorem 4.2 and the universal coefficient theorem imply that $H_{3}(\mathcal{J} \otimes$ $\mathbb{Z} / 2)$ has dimension $\operatorname{dim} H_{1}\left(\partial K_{0}\right)+2 \operatorname{dim} H_{1}(K)$, so we must show $\operatorname{dim} H_{1}\left(K_{0}\right)=$ $(1 / 2) \operatorname{dim} H_{1}\left(\partial K_{0}\right)+\operatorname{dim} H_{1}(K)$. The long exact sequence for $\left(K, K-K_{0}\right)$ and excision show $H_{2}\left(K, K_{0}\right)=H_{1}\left(\partial K_{0}\right)$, and inserting this in the long exact sequence for $\left(K, K_{0}\right)$ gives an exact sequence

$$
H_{1}\left(\partial K_{0}\right) \stackrel{i_{*}}{\rightarrow} H_{1}\left(K_{0}\right) \stackrel{j_{*}}{\rightarrow} H_{1}(K) \rightarrow 0,
$$

where the maps are the natural maps. Since $\operatorname{dim} \operatorname{Ker}\left(i_{*}\right)=(1 / 2) \operatorname{dim} H_{1}\left(\partial K_{0}\right)$ by Poincaré duality, the desired equality follows.

Note that the above sequence induces a short exact sequence

$$
0 \rightarrow \mathcal{K} \stackrel{i_{*}}{\rightarrow} H_{1}\left(K_{0}\right) \stackrel{j_{*}}{\rightarrow} H_{1}(K) \rightarrow 0,
$$


with $\mathcal{K}=H_{1}\left(\partial K_{0}\right) / \operatorname{Ker}\left(i_{*}\right)$.

Step 3. (Coefficients are still $\mathbb{Z} / 2$.) There is a commutative diagram with exact rows and columns:

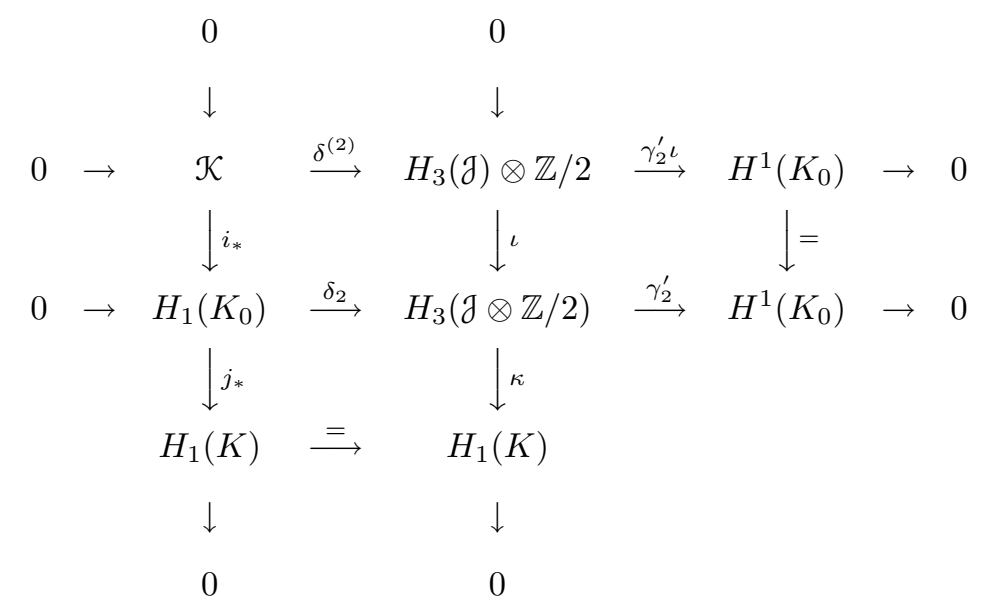

Here the middle vertical sequence is the universal coefficient theorem for the chain complex $\mathcal{J}$ and the middle horizontal sequence is the sequence of Step 2. We must describe the top horizontal sequence and prove commutativity of the diagram; exactness of the top sequence then follows by a diagram chase.

The map $\delta^{(2)}$ is induced by the map $\delta \otimes \mathbb{Z} / 2: H_{1}\left(\partial K_{0}\right) \rightarrow H_{3}(\mathcal{J}) \otimes \mathbb{Z} / 2$ : indeed, $\iota(\delta \otimes \mathbb{Z} / 2)=\delta_{2} i_{*}$ (this was pointed out at the beginning of the proof of Theorem 5.1), so $\delta \otimes \mathbb{Z} / 2$ has kernel $\operatorname{Ker}\left(i_{*}\right)$, so $\delta^{(2)}$ can be defined to make the upper left square commute.

It remains to show commutativity of the lower square. For $a \in H_{3}(\mathcal{J} \otimes \mathbb{Z} / 2)$ one computes $\kappa(a)$ as follows. Represent $a$ by an element $A \in J$. Then $\beta^{*}(A)$ is 0 modulo 2. The desired element $\kappa(a) \in H_{2}(\mathcal{J})=H_{1}(K)$ is represented by $(1 / 2) \beta^{*}(A)$.

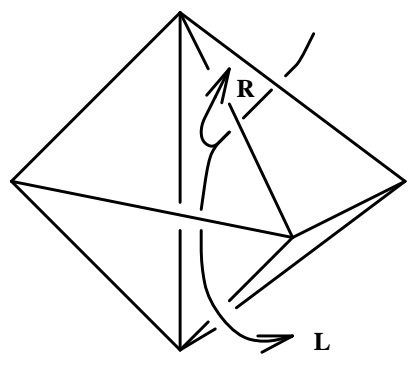

Figure 14

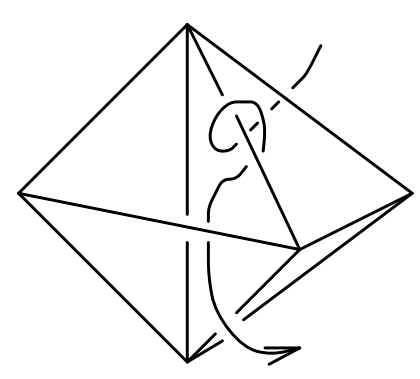

Figure 15

Consider a general path $C$ in $K_{0}$. As $C$ passes through a 3-simplex $\Delta$ it determines an edge $e$ of $\Delta$, as previously described, and $\delta_{2}(C)$ is represented by the sum of the elements of $J$ corresponding to these $e$. Call the edge $E$ of $K$ corresponding to $e$ the "axis" of $C$ passing through $\Delta$. In the next 3 -simplex $C$ may continue around 


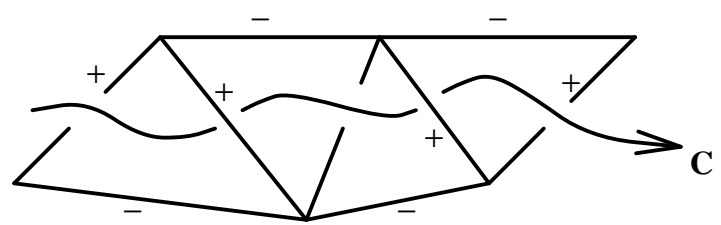

Figure 16

the same axis, turn left, or turn right (see Fig. 14). We can eliminate left turns from the path $C$ without changing its homology class, as illustrated in Fig. 15. Thus, without loss of generality, $C$ has no left turns. The successive axes of $C$ then form a path in $K$ homologous to $C$. Moreover, each pair of successive axes determines a face of a 3 -simplex in $K$ and hence an additional edge (the third edge of that face). By inspection, one sees that $\kappa \delta_{2}(C)$ is represented by the sum of the axes minus the sum of these additional edges (Fig. 16), and this is homologous to $C$ modulo 2, as desired.

Step 4. (Omitted coefficients are still $\mathbb{Z} / 2$.) There is a commutative diagram with exact rows and columns:

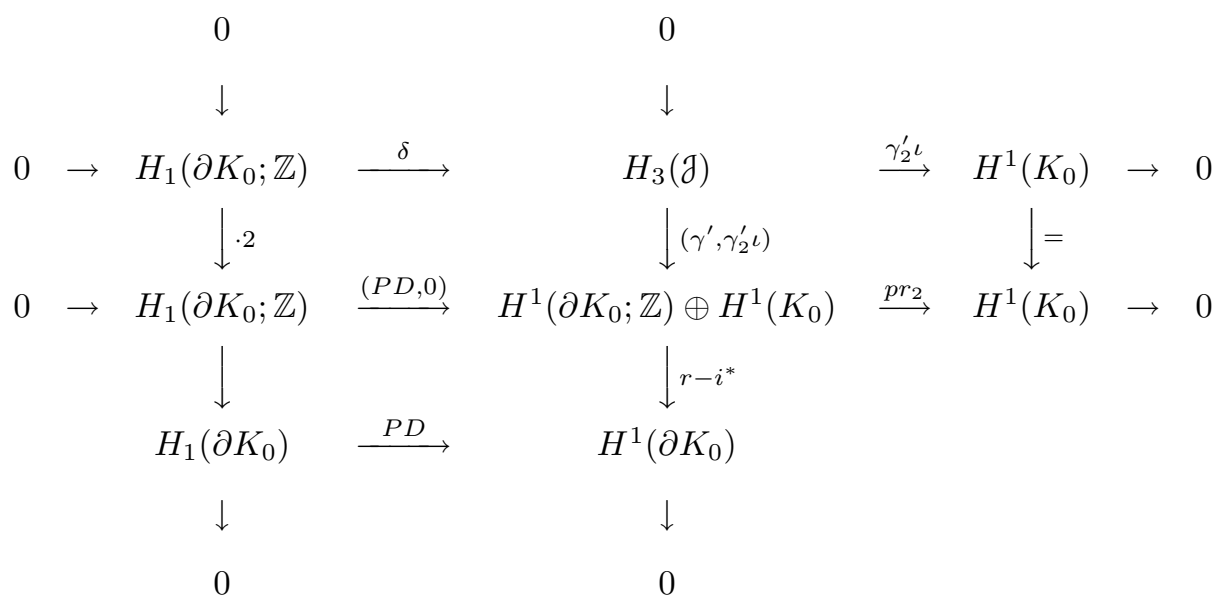

The commutativity of the top left square is by definition of $\gamma^{\prime}$ and by Lemma 4.3, and the commutativity of the rest of the diagram is trivial. Exactness of the top row follows directly from exactness of the top row of the previous diagram plus the fact (Lemma 4.3 and Theorem 4.2) that $\delta$ is injective with 2-torsion cokernel. Exactness of the second row and first column is trivial. Exactness of the middle column now follows by a diagram chase, completing the proof of Step 4 and of Theorem 5.1. 


\section{Proof of Theorem 2.4}

Let $\bar{J}_{\Delta}$ be defined like $J_{\Delta}$ but without the relation $e_{1}+e_{2}+e_{3}=0$; that is, $\bar{J}_{\Delta}$ is generated by the six edges $e_{1}, \ldots, e_{6}$ of $\Delta$ with the relations $e_{i}=e_{i+3}$ for $i=1,2,3$. We give $\bar{J}_{\Delta}$ the standard bilinear form $\left(e_{i}, e_{j}\right)=\delta_{i j}$ for $i, j \in\{1,2,3\}$. Let $\bar{J}$ be the orthogonal sum of the $\bar{J}_{\Delta}$. The map $\beta^{*}: J \rightarrow C_{2}$ can be factored as

$$
\beta^{*}: J \stackrel{\beta_{1}}{\longrightarrow} \bar{J} \stackrel{\beta_{2}}{\longrightarrow} C_{2}
$$

with $\beta_{1}$ and $\beta_{2}$ defined on the $\Delta$-component by

$$
\left.\begin{array}{l}
\beta_{1}\left(e_{i}\right)=e_{i+1}-e_{i+2} \\
\beta_{2}\left(e_{i}\right)=j\left(e_{i}\right)+j\left(e_{i+3}\right)
\end{array}\right\} \text { for } i=1,2,3
$$

Note that $\beta_{1}: J \rightarrow \bar{J}$ and the natural projection $p: \bar{J} \rightarrow J$ are adjoint maps with respect to the forms on $J$ and $\bar{J}$; that is,

$$
\left(\beta_{1}(a), x\right)=\langle a, p(x)\rangle \quad \text { for } a \in J \text { and } x \in \bar{J} .
$$

Let $C$ be a general closed path in $\partial K_{0}$ with no back-tracking. As described in Sect. 4, as $C$ passes through a 2-simplex of $\partial K_{0}$ it determines an edge $e$ of the corresponding 3 -simplex $\Delta$ and a sign \pm , and hence an element-which we call $\pm e-$ of $\bar{J}$. Let $\bar{\delta}(e)$ be the sum of all these $\pm e$. Thus the map $\delta: H_{1}\left(\partial K_{0}\right) \rightarrow H_{3}$ of Sects. 4 and 5 is given by

$$
\delta([C])=[p \bar{\delta}(C)] .
$$

For $x \in \bar{J}$ define an integer-valued map $\bar{\gamma}(x)$ on the set of closed non-back-tracking paths on $\partial K_{0}$ by

$$
\bar{\gamma}(x)(C)=(x, \bar{\delta}(C)) .
$$

(Note that $\bar{\gamma}(x)(C)$ will usually not just depend on the homology class of $C$.) The map $\gamma^{\prime}$ of the previous section is given by

$$
\gamma^{\prime}(a)=\bar{\gamma}\left(\beta_{1}(a)\right) \text { for } a \in J,
$$

since, by (6.4), (6.2), (6.3), and the definition of $\gamma^{\prime}, \bar{\gamma}\left(\beta_{1}(a)\right)(C)=\left(\beta_{1}(a), \bar{\delta}(C)\right)=$ $\langle a, \delta(C)\rangle=\gamma^{\prime}(a)(C)$.

Similarly, if $C$ is a general closed path with no back-tracking in $K$, then as $C$ passes through a 3 -simplex $\Delta$ of $K$ it determines an edge $e$ of $\Delta$ (the edge common to the two faces that it passes through), and we define $\bar{\delta}_{2}(C) \in \bar{J} \otimes \mathbb{Z} / 2$ to be the modulo 2 sum of these $e$. For $x \in \bar{J}$ define a $\mathbb{Z} / 2$-valued map $\bar{\gamma}_{2}(x)$ on the set of general closed non-back-tracking paths in $K$ by

$$
\bar{\gamma}_{2}(x)(C)=\left(x, \bar{\delta}_{2}(C)\right) \quad(\bmod 2) .
$$

As for $\bar{\gamma}$ one sees that the map $\gamma_{2}^{\prime}$ of the previous section is given by

$$
\gamma_{2}^{\prime}(a)=\bar{\gamma}_{2} \beta_{1}(a) \quad \text { for } a \in J .
$$


Lemma 6.1. Suppose every component of $\partial K_{0}$ is a torus. Then there exists an element $\eta \in \bar{J}$ satisfying:

1. the $\bar{J}_{\Delta}$ component of $\eta$ has coefficient sum 1 for each 3-simplex $\Delta$,

2. $\beta_{2}(\eta)=2 \sum E$, twice the sum of all the edges of $K$,

3. $\bar{\gamma}(\eta)(C)=0$ for every general essential simple closed curve $C$ in $\partial K_{0}$ with no back-tracking,

4. $\bar{\gamma}_{2}(\eta)(C)=0$ modulo 2 for every general closed curve in $K_{0}$ with no backtracking.

Moreover, any such $\eta$ is unique up to $\operatorname{Im} \beta_{1} \beta$.

Proof. Choose any element $x$ of $\bar{J}$ which satisfies condition 1 . The contribution at a vertex $V$ of $K$ to $\alpha^{*}\left(\beta_{2}(x)-2 \sum E\right)$ is $n_{2}(V)-2 n_{0}(V)$, where $n_{i}(V)$ is the number of $i$-simplices in the link $L_{V}$ of vertex $V$. But $n_{2}(V)-2 n_{0}(V)=-2 \chi\left(L_{V}\right)$, which is zero since $L_{V}$ is a torus. Thus $\beta_{2}(x)-2 \sum E$ is in $\operatorname{Ker} \alpha^{*}$.

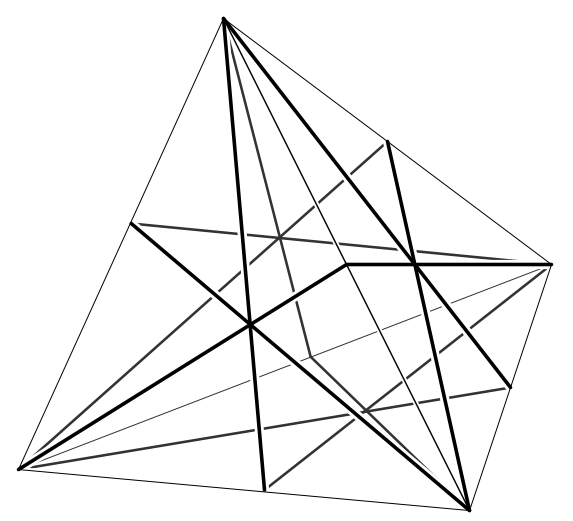

Figure 17

We claim that $\beta_{2}(x)-2 \sum E$ is in $\operatorname{Im} \beta^{*}$, that is, it represents zero in $H_{2}(\mathcal{J})=$ $H_{1}(K ; \mathbb{Z} / 2)$. Indeed, let $x_{\Delta}$ be the $\bar{J}_{\Delta}$-component of $x$ and consider $\beta_{2}\left(x_{\Delta}\right)$ as a modulo-2 1-chain in $\Delta \subseteq K$. The boundary of this 1-chain is the sum of the vertices of $\Delta$. The same is true for the 1-chain $S_{\Delta}$ pictured in Fig. 17 (the edges of the tetrahedron are not part of the chain). Hence $\beta_{2}\left(x_{\Delta}\right)-S_{\Delta}$ is a 1-cycle in $\Delta \subseteq K$, hence a 1boundary. Thus, as a modulo-2 1-cycle, $\beta_{2}(x)$ differs from $\sum S_{\Delta}$ by a boundary. Since $\sum S_{\Delta}$ is identically zero modulo $2, \beta_{2}(x)-2 \sum E$ is a modulo- 2 boundary, as claimed.

Now choose $a \in J$ with $\beta^{*}(a)=\beta_{2}(x)-2 \sum E$. Then $y=x-\beta_{1}(a)$ satisfies conditions 1 and 2 of the lemma.

Now it is not hard to verify, given that $y$ satisfies conditions 1 and 2 of the lemma, that for a general essential simple closed curve $C$ in $\partial K_{0}$ without back-tracking, $\bar{\gamma}(y)(C)$ only depends on the homology class of $C$. Moreover, if one restricts to curves of this type (or more generally, to general curves $C$ without back-tracking that are isotopic through immersed curves to essential simple closed curves, i.e., $C$ should have no "winding"), the map $C \mapsto \bar{\gamma}(y)(C)$ defines a homomorphism $H^{1}\left(\partial K_{0}\right) \rightarrow \mathbb{Z}$, and hence an element $c$ of $H^{1}\left(\partial K_{0}\right)$. Similarly, evaluation of $\bar{\gamma}_{2}$ on general closed curves in $K_{0}$ without 
back-tracking defines an element $c_{2} \in H^{1}\left(K_{0} ; \mathbb{Z} / 2\right)$. Moreover, $r(c)=i^{*}\left(c_{2}\right)$. Thus, by Theorem 5.1, there exists a $w \in H_{3}(\mathcal{J})$ with $\left(\gamma^{\prime}(w), \gamma_{2}^{\prime} \iota(w)\right)=\left(c, c_{2}\right)$. Let $a \in J$ be a representative for $w$. Then $\eta=y-\beta_{1}(a)$ satisfies conditions $1,2,3$, and 4 .

Given two elements $\eta$ satisfying the conditions, their difference $\zeta$ will satisfy:

$1^{\prime}$. The $\bar{J}_{\Delta}$ of $\zeta$ has coefficient sum 0 for each 3 -simplex $\Delta$,

$2^{\prime} . \beta_{2}(\zeta)=0$,

and conditions 3 and 4 of the lemma. Condition $1^{\prime}$ is equivalent to $\zeta \in \operatorname{Im} \beta_{1}$, say $\zeta=\beta_{1}(c)$. Condition $2^{\prime}$ then says that $c \in \operatorname{Ker} \beta^{*}$, so $c$ is a cycle for $H_{3}(\mathcal{J})$. Conditions 3 and 4 say that $c$ represents 0 in $H_{3}(\mathcal{J})$, so $c$ is in $\operatorname{Im} \beta$.

Note that for any $\eta$ satisfying condition 1 of the lemma one can solve equations (2.8) for $c_{\nu}^{\prime}$ and $c_{\nu}^{\prime \prime}$. Theorem 2.4 is thus just a restatement of the above lemma, so it is proved.

\section{References}

[CS] M. Culler and P. B. Shalen, Varieties of group representations and splittings of 3-manifolds, Ann. of Math. 117 (1983), 109-146

[D] Johan L. Dupont, The dilogarithm as a characteristic class for flat bundles, Proc. of the Northwestern conference on cohomology of groups (Evanston, Ill., 1985), J. Pure Appl. Algebra 44 (1987), 137-164.

[HMW] Craig D. Hodgson, G. Robert Meyerhoff, and Jeffrey R. Weeks, Surgeries on the Whitehead link yield geometrically similar manifolds, These Proceedings.

[MN] R. Meyerhoff and Walter D. Neumann, An asymptotic formula for the eta invariants of hyperbolic 3-manifolds, to appear in Comment. Math. Helvetici (1991).

[M] R. Meyerhoff, The Chern-Simons invariant for hyperbolic 3-manifolds, Thesis, Princeton 1981.

[NR] W. D. Neumann and Alan W. Reid, Arithmetic of hyperbolic 3-manifolds, These Proceedings.

[NZ] Walter D. Neumann and Don Zagier, Volumes of hyperbolic three-manifolds, Topology 24 (1985), 307-332.

[Y1] Tomoyoshi Yoshida, The $\eta$-invariant of hyperbolic 3-manifolds, Invent. Math. 81 (1985), 473-514

[Y2] Tomoyoshi Yoshida, On ideal points of deformation curves of hyperbolic 3-manifolds with one cusp, To appear in Topology

Department of Mathematics, The Ohio State University, Columbus, $\mathrm{OH} 43210$

Email: neumann@mps.ohio-state.edu 